\title{
Tracking the variable jets of V404 Cygni during its 2015 outburst
}

\author{
A. J. Tetarenko ${ }^{\oplus,}, 2 \star$ G. R. Sivakoff, ${ }^{1}$ J. C. A. Miller-Jones ${ }^{\oplus},{ }^{3}$ M. Bremer, ${ }^{4}$ \\ K. P. Mooley ${ }^{\oplus}, 5,6,7$ R. P. Fender, ${ }^{5}$ C. Rumsey, ${ }^{8}$ A. Bahramian ${ }^{\oplus}, 3$ \\ D. Altamirano, ${ }^{9}$ S. Heinz, ${ }^{10}$ D. Maitra, ${ }^{11}$ S. B. Markoff, ${ }^{12}$ S. Migliari, ${ }^{13,14}$ \\ M. P. Rupen, ${ }^{6,15}$ D. M. Russell ${ }^{\odot},{ }^{16}$ T. D. Russell ${ }^{\oplus 12}$ and C. L. Sarazin ${ }^{17}$ \\ ${ }^{1}$ Department of Physics, University of Alberta, CCIS 4-181, Edmonton, AB T6G 2E1, Canada \\ ${ }^{2}$ East Asian Observatory, 660 N. A'ohoku Place, University Park, Hilo, HI 96720, USA \\ ${ }^{3}$ International Centre for Radio Astronomy Research-Curtin University, GPO Box U1987, Perth, WA 6845, Australia \\ ${ }^{4}$ Institute de Radioastronomie Millimétrique, 300 Rue de la Piscine, 38406 Saint Martin d'Hères, France \\ ${ }^{5}$ Department of Physics, University of Oxford, Keble Road, Oxford OX1 3RH, UK \\ ${ }^{6}$ National Radio Astronomy Observatory, Socorro, NM 87801, USA \\ ${ }^{7}$ Caltech, 1200 E. California Blvd. MC 249-17, Pasadena, CA 91125, USA \\ ${ }^{8}$ Astrophysics Group, Cavendish Laboratory, $19 \mathrm{~J}$. J. Thomson Avenue, Cambridge CB3 OHE, UK \\ ${ }^{9}$ School of Physics and Astronomy, University of Southampton, Highfield, Southampton SO17 1BJ, UK \\ ${ }^{10}$ Astronomy Department, University of Wisconsin-Madison, 475. N. Charter St., Madison, WI 53706, USA \\ ${ }^{11}$ Department of Physics and Astronomy, Wheaton College, Norton, MA 02766, USA \\ ${ }^{12}$ Anton Pannekoek Institute for Astronomy, University of Amsterdam, PO Box 94249, NL-1090 GE Amsterdam, the Netherlands \\ ${ }^{13}$ Institute of Cosmos Sciences, University of Barcelona, Martí i Franquès 1, E-08028 Barcelona, Spain \\ ${ }^{14}$ XMM-Newton Science Operations Centre, ESAC/ESA, PO Box 78, E-28691 Villanueva de la Cañada, Madrid, Spain \\ ${ }^{15}$ National Research Council, Herzberg Astronomy and Astrophysics, 717 White Lake Road, PO Box 248, Penticton, BC V2A 6J9, Canada \\ ${ }^{16}$ New York University Abu Dhabi, PO Box 129188, Abu Dhabi, United Arab Emirates \\ ${ }^{17}$ Department of Astronomy, University of Virginia, PO Box 400325, Charlottesville, VA 22904, USA
}

Accepted 2018 October 12. Received 2018 October 10; in original form 2018 June 8

\begin{abstract}
We present multifrequency monitoring observations of the black hole X-ray binary V404 Cygni throughout its 2015 June outburst. Our data set includes radio and $\mathrm{mm} / \mathrm{sub}-\mathrm{mm}$ photometry, taken with the Karl G. Jansky Very Large Array, Arc-Minute MicroKelvin Imager Large Array, Sub-millimeter Array, James Clerk Maxwell Telescope, and the Northern Extended Millimetre Array, combined with publicly available infrared, optical, UV, and X-ray measurements. With these data, we report detailed diagnostics of the spectral and variability properties of the jet emission observed during different stages of this outburst. These diagnostics show that emission from discrete jet ejecta dominated the jet emission during the brightest stages of the outburst. We find that the ejecta became fainter, slower, less frequent, and less energetic, before the emission transitioned (over 1-2 d) to being dominated by a compact jet, as the outburst decayed towards quiescence. While the broad-band spectrum of this compact jet showed very little evolution throughout the outburst decay (with the optically thick to thin synchrotron jet spectral break residing in the near-infrared/optical bands; $\sim 2-5 \times 10^{14} \mathrm{~Hz}$ ), the emission still remained intermittently variable at $\mathrm{mm} / \mathrm{sub}-\mathrm{mm}$ frequencies. Additionally, we present a comparison between the radio jet emission throughout the 2015 and previous 1989 outbursts, confirming that the radio emission in the 2015 outburst decayed significantly faster than in 1989. Lastly, we detail our sub-mm observations taken during the 2015 December mini-outburst of V404 Cygni, which demonstrate that, similar to the main outburst, the source was likely launching jet ejecta during this short period of renewed activity.
\end{abstract}

Key words: black hole physics - stars: individual (V404 Cygni, GS 2023+338)-ISM: jets and outflows - radio continuum: stars - submillimetre: stars - X-rays: binaries. 


\section{INTRODUCTION}

Black hole X-ray binaries (BHXBs) contain a stellar mass black hole accreting matter from a companion star, where a portion of the accreted material can be transported back outwards in the form of a relativistic jet. These systems are typically transient in nature, evolving from periods of inactivity into a bright out-bursting state lasting days to months. BHXB jet emission can span many decades in frequency, and during these outbursts, the intensity, morphology, spectral, and temporal properties of the jet emission are known to vary with accretion state (Fender, Belloni \& Gallo 2004; Belloni 2010).

In quiescence and the hard accretion state, there exists a steady, compact synchrotron-emitting jet, which primarily emits at radio, sub-mm, and optical/infrared (OIR) frequencies (Fender 2001; Corbel \& Fender 2002; Gallo, Fender \& Hynes 2005; Russell et al. 2006, 2013b; Chaty, Dubus \& Raichoor 2011; Plotkin, Gallo \& Jonker 2013; Plotkin et al. 2015, 2016; Tetarenko et al. 2015a). This compact jet displays a characteristic flat to slightly inverted broad-band spectrum ( $\alpha \geq 0, f_{v} \propto v^{\alpha}$; Blandford \& Königl 1979; Falcke \& Biermann 1995; Fender 2001) extending up to OIR frequencies (Corbel \& Fender 2002; Casella et al. 2010; Chaty et al. 2011), where it breaks to an optically thin spectrum $(\alpha \sim-0.7$; Russell et al. 2013a). The location of the spectral break marks the most compact region of the jet, where particles are first accelerated to high energies (Markoff, Falcke \& Fender 2001; Markoff, Nowak \& Wilms 2005; Chaty et al. 2011), and has only been directly observed in a few BHXBs ( $\nu_{\text {break }} \sim 10^{11-14} \mathrm{~Hz}$; e.g. Russell et al. 2013a,b).

As the system evolves through the rising hard state, where the $\mathrm{X}$-ray luminosity (and, in turn the mass accretion rate) increases, the compact jet spectrum also evolves. In particular, the location of the spectral break has been observed to shift to lower frequencies (towards the radio regime), as the source transitions into a softer accretion state (van der Horst et al. 2013; Russell et al. 2014). This spectral evolution cannot be driven solely by optical depth effects, which predict an opposite scaling for the spectral break frequency $\left(v_{\text {break }} \propto \dot{M}^{2 / 3}\right.$; Falcke \& Biermann 1995). Alternatively, recent work (Koljonen et al. 2015), which shows a correlation between the location of the spectral break and the photon index of the $\mathrm{X}$-ray spectrum, suggests that the particle acceleration properties within jets (traced by the flux density and frequency of the spectral break) may instead be connected to the properties of the plasma close to the black hole.

During the transition between the hard and soft accretion states, the jet emission can switch from being dominated by a compact jet to arising from discrete jet ejections (e.g. Mirabel \& Rodríguez 1994; Hjellming \& Rupen 1995; Kuulkers et al. 1999; Corbel et al. 2002; Miller-Jones et al. 2012; Brocksopp et al. 2013; Tetarenko et al. 2017). These ejecta have an optically thin spectrum $(\alpha<0)$ above the self-absorption/free-free absorption turnover frequency (MillerJones et al. 2004), display highly variable emission, and their expansion/bulk motion can be resolved and tracked with Very Long Baseline Interferometry (VLBI; e.g. Hjellming \& Rupen 1995; Tingay et al. 1995; Miller-Jones et al. 2004). Once the source reaches the soft state, jet emission is believed to be quenched altogether (or faint enough to be below the detection thresholds of current instruments; Fender et al. 1999; Corbel et al. 2001; Coriat et al. 2011; Russell et al. 2011; Rushton et al. 2016), with any residual radio emission usually attributed to an interaction between the jet ejecta and the surrounding medium (e.g. Corbel et al. 2004).

By tracking spectral, temporal, and morphological changes in the jet emission over an outburst, physical conditions in the jet can be linked to the properties of the accretion flow (probed at X-ray frequencies), potentially revealing which accretion flow properties govern the launching, evolution, and quenching of jets (Russell et al. 2013b, 2014, 2015). Therefore, multifrequency studies of these jets during outburst, which track jet emission properties at different physical scales along the jet axis, are essential in understanding the mechanisms that govern BHXB jet behaviour. Within the BHXB population, V404 Cygni (aka GS 2023 + 338; hereafter referred to as V404 Cyg) is an optimal candidate for multifrequency jet studies due to its proximity (2.39 $\pm 0.14 \mathrm{kpc}$; Miller-Jones et al. 2009), low extinction $[E(B-V)=1.3$; Casares et al. 1993], and high X-ray luminosity levels in outburst $\left(L_{X} \sim 1 \times 10^{39} \mathrm{erg} \mathrm{s}^{-1}\right.$; Motta et al. $2017 \mathrm{~b})$ and quiescence $\left(L_{X} \sim 1 \times 10^{33} \mathrm{erg} \mathrm{s}^{-1}\right.$; Corbel, Kording \& Kaaret 2008).

V404 Cyg was first discovered in outburst in 1989 (Makino 1989), after which it remained in a low-luminosity quiescent state for $\sim 26$ yr. During this prolonged quiescent state, V404 Cyg displayed a spectrum that was measured to be flat across the radio band ( $\alpha=$ $-0.05 \pm 0.15$, where $f_{v} \propto v^{\alpha}$ and $\left.v=1.4-8.4 \mathrm{GHz}\right)$, likely extending up to IR frequencies, where it breaks to an optically thin spectrum ( $\alpha<0$; Hynes et al. 2009). This radio spectrum is consistent with originating from a partially self-absorbed synchrotron jet (Blandford \& Königl 1979). While optical and UV emissions in the quiescent spectrum of V404 Cyg are well described by blackbody emission from the known K0IV companion star, Muno \& Mauerhan (2006) report a mid-IR $(4.5$ and $8 \mu \mathrm{m})$ excess above the level expected from the companion star. This mid-IR excess could originate from the accretion disc (Muno \& Mauerhan 2006; Hynes et al. 2009), the compact jet (Gallo et al. 2007), or a combination of the two. The average radio flux density of the jet in quiescence is $\sim 0.3 \mathrm{mJy}$ (Gallo, Fender \& Pooley 2003a), although the emission is known to be highly variable (Hynes et al. 2009; Plotkin et al. in preparation), reaching up to $\sim 1.5 \mathrm{mJy}$ (Hjellming et al. 2000). The quiescent jet of V404 Cyg is unresolved with the global VLBI array, but Miller-Jones et al. (2009) placed an upper limit on the compact jet size scale of $<1.4$ au at $22 \mathrm{GHz}$.

During its discovery outburst in 1989, V404 Cyg displayed bright $\mathrm{X}$-ray flaring activity. This highly variable emission was found to not always be intrinsic to the source, but at times be caused by large changes in column density, where the accretion flow became obscured (Terada et al. 1994; Oosterbroek et al. 1997; Zycki, Done \& Smith 1999b). V404 Cyg displayed a variety of radio behaviour during this outburst (Han \& Hjellming 1992), where the radio spectrum evolved from steep $(\alpha<0)$ to inverted $(\alpha>0)$ in a matter of days. Further, significant radio flux variability on time-scales as short as tens of minutes was observed, and there were hints of coupled radio, optical, and X-ray emissions. However, the instruments available during this 1989 outburst did not have the capabilities to perform the simultaneous, multifrequency, time-resolved observations needed to fully understand this rapidly evolving jet source.

In 2015 June, V404 Cyg entered a new outburst (Barthelmy et al. 2015; Negoro et al. 2015; Kuulkers et al. 2015; Bernardini et al. 2016), providing a unique opportunity to study the evolving jet with observational coverage that was not possible during the 1989 outburst. During this new outburst, V404 Cyg exhibited bright multifrequency variability, in the form of large-amplitude flaring events (e.g. Ferrigno et al. 2015; Gandhi et al. 2015; Gazeas et al. 2015; Mooley et al. 2015; Motta et al. 2015a,b; Tetarenko et al. 2015b,c), for $\mathrm{a} \sim 2$ week period, before the flaring activity ceased at all wavelengths, and the source began to decay (Sivakoff et al. 2015a,b; Plotkin et al. 2017a) back towards quiescence. Additionally, in late 2015 December V404 Cyg entered a short mini-outburst period, 
during which it displayed renewed flaring activity (e.g. Beardmore, Page \& Kuulkers 2015; Lipunov et al. 2015; Malyshev et al. 2015; Trushkin, Nizhelskij \& Tsybulev 2015; Motta et al. 2016; Tetarenko et al. 2016; Muñoz-Darias et al. 2017; Kajava et al. 2018).

In this paper, we present multifrequency monitoring of V404 Cyg during this 2015 outburst, including radio and $\mathrm{mm} / \mathrm{sub}-\mathrm{mm}$ photometry, combined with publicly available OIR, UV, and Xray measurements. Radio frequency data were taken with NSF's Karl G. Jansky Very Large Array (VLA) and the Arc-Minute MicroKelvin Imager Large Array (AMI-LA), while the mm/sub-mm frequency data were taken with the Sub-millimeter Array (SMA), the Sub-millimetre Common User Bolometric Array-2 instrument on the James Clerk Maxwell Telescope (JCMT SCUBA-2), and the Institute de Radioastronomie Millimétrique's Northern Extended Millimetre Array (IRAM NOEMA). Our observations span a time period from hours after the initial X-ray detection of the outburst, until late in its decay back towards quiescence. While our team's earlier work (Tetarenko et al. 2017) probed the jet emission during a portion of the brightest flaring period (on 2015 June 22) of the outburst, this work aims to track the spectral and temporal changes in the jet emission as the system transitioned away from the flaring state and began to decay back into quiescence. In Section 2, we describe the data collection and data reduction processes. In Section 3, we present multifrequency light curves and broad-band spectra. In Section 4, we use this series of observations to discuss the jet properties in V404 Cyg, as well as draw comparisons to the previous 1989 outburst, and the December 2015 mini-outburst. A summary of our work is presented in Section 5.

\section{OBSERVATIONS AND DATA ANALYSIS}

\subsection{VLA radio frequency observations}

We observed V404 Cyg with the VLA (project codes 15A-504 and 15A-509) from 2015 July 02 to July 12 (MJD 57205-57215) in the $L$ (1-2 GHz), C (4-8 GHz), Ku (12-18 GHz), and $K(18-26 \mathrm{GHz})$ bands. The array was in its most extended A-configuration for all observations, where we split the array into two or three sub-arrays to obtain strictly simultaneous observations across multiple bands. All observations were made with the 8-bit samplers, generating two base-bands, each with eight spectral windows of $642-\mathrm{MHz}$ channels, giving a total bandwidth of $1.024 \mathrm{GHz}$ per base-band (see Table A1 for a summary of the array set-up of all the observations). Flagging, calibration, and imaging (with natural weighting chosen to maximize sensitivity) of the data were carried out within the Common Astronomy Software Application package (CASA v4.3.1; McMullin et al. 2007) using standard procedures outlined in the CASA Guides ${ }^{1}$ for VLA data reduction (i.e. a priori flagging, setting the flux density scale, initial phase calibration, solving for antennabased delays, bandpass calibration, gain calibration, scaling the amplitude gains, and final target flagging). We used J2025+3343 as a phase calibrator for all epochs, and 3C $48(0137+331)$ as a flux calibrator in all epochs but July 11 (MJD 57214), where 3C 147 $(0542+498)$ was used. When imaging the lower-frequency bands (1-2 and 4-8 GHz), we placed outlier fields on other bright sources within the primary beam to ensure that their side-lobes did not affect our flux density measurement of V404 Cyg. Flux densities of the source were measured by fitting a point source in the image plane (using the imf it task) and, as is standard for VLA L/C/Ku/K band

${ }^{1}$ https://casaguides.nrao.edu data, systematic errors of $1 / 1 / 3 / 3$ per cent were added (Perley \& Butler 2017). All VLA flux density measurements are reported in Table B1. Given the rapidly changing radio flux density observed in this outburst, we also imaged the source on shorter time-scales (less than the full observation period), using our custom CASA variability measurement scripts ${ }^{2}$ (see section 3.1 of Tetarenko et al. 2017 for a detailed description of the capabilities of these scripts).

\subsection{AMI-LA radio frequency observations}

V404 Cyg was observed with the AMI-LA (Zwart et al. 2008) radio telescope throughout the 2015 outburst. Observations were carried out with the analogue lag correlator using six frequency channels spanning 13.5-18.0 GHz. The raw data were processed (RFI excision and calibration) with a fully automated pipeline, AMI-REDUCE (e.g. Davies et al. 2009; Perrott et al. 2013). Daily measurements of 3C 48 and 3C 286 were used for the absolute flux calibration, which is good to about 10 per cent. The calibrated and RFI-flagged data were then imported into CASA for imaging. In this paper, we use a sub-set of the AMI-LA observations taken during this outburst (complete data set will be published in Fender et al. in preparation). Our analysis includes AMI-LA data that were taken simultaneously with our NOEMA mm/sub-mm observations; from 2015 June 2630 and July 11-12.

\subsection{NOEMA (sub)-mm frequency observations}

We observed V404 Cyg with the NOEMA (project codes S15DE and D15AB) between 2015 June 26 and July 13 (MJD 57199-57216) in the $3 \mathrm{~mm}$ (tuning frequency of $97.5 \mathrm{GHz}$ ) and $2 \mathrm{~mm}$ (tuning frequency of $140 \mathrm{GHz}$ ) bands. These observations were made with the WideX correlator, to yield 1 base-band, with a total bandwidth of $3.6 \mathrm{GHz}$ per polarization (see Table A2 for a summary of the correlator and array set-up of all the observations). We used J2023+336 as a phase calibrator, and MWC349 as a flux calibrator, in all epochs. The bandpass calibrator varied between epochs: 3C 273 (57199 at 3mm), 3C 454.3 (57200/57203 at $2 \mathrm{~mm}$ ), J1749 + 096 (57202 at $3 \mathrm{~mm}$ and 57200/57201 at $2 \mathrm{~mm}$ ), and $\mathrm{J} 2013+370(57215 / 57216$ at 2 and $3 \mathrm{~mm})$. As CASA is unable to handle NOEMA data in its original format, flagging and calibration of the data were first performed in GILDAS ${ }^{3}$ using standard procedures, then the data were exported to $\mathrm{CASA}^{4}$ for imaging (with natural weighting to maximize sensitivity). Flux densities of the source were measured by fitting a point source in the image plane (using the imfit task). All NOEMA flux density measurements can be seen in Table B2. Given the rapidly changing (sub)-mm flux density observed in this outburst, we also imaged the source on shorter time-scales (less than the full observation period), using our custom CASA variability measurement scripts.

\subsection{SMA (sub)-mm frequency observations}

We observed V404 Cyg with the SMA (project code 2015A-S026) between 2015 June 16 and July 02 (MJD 57189-57215). All of our

\footnotetext{
${ }^{2}$ These scripts are publicly available on github; https://github.com/Astroua /AstroCompute_Scripts.

${ }^{3}$ http://www.iram.fr/IRAMFR/GILDAS

${ }^{4}$ To convert a NOEMA data set for use in CASA, we followed the procedures outlined at https://www.iram.fr/IRAMFR/ARC/documents/filler/casa-gilda s.pdf.
} 
observations utilized the ASIC and/or SWARM correlators, tuned to an LO frequency of $224 \mathrm{GHz}$ (see Table A3 for a summary of the correlator and array set-up of all the observations). We performed all flagging, calibration, and imaging (with natural weighting to maximize sensitivity) of the data within CASA, using the same procedures and calibrators outlined in section 2.2 of Tetarenko et al. (2017). Flux densities of the source were measured by fitting a point source in the image plane (using the imfit task). All SMA flux density measurements are reported in Table B2. Given the rapidly changing (sub)-mm flux density observed in this outburst, we also imaged the source on shorter time-scales (less than the full observation period), using our custom CASA variability measurement scripts.

\subsection{JCMT SCUBA-2 (sub)-mm frequency observations}

We observed V404 Cyg with the JCMT (project code M15AI54) on 2015 June 17 and July 02 (MJDs 57190 and 57205) in the $850 \mu \mathrm{m}(350 \mathrm{GHz})$ and $450 \mu \mathrm{m}(666 \mathrm{GHz})$ bands. On June 17, the observation consisted of five $\sim 30$ min scans on target with the SCUBA-2 detector (Chapin et al. 2013; Holland et al. 2013) from 11:13:12-14:19:05 UTC (MJD 57190.468-57190.597). On July 02 , the observation consisted of eight $\sim 30 \mathrm{~min}$ scans on target with the SCUBA-2 detector from 09:01:23-13:42:29 UTC (MJD 57205.376 - 57205.571). During the observations on June 17 , we were in the Grade 4 weather band with a $225 \mathrm{GHz}$ opacity of $0.1-0.2$, while on July 02 we were in the Grade 3 weather band with a $225 \mathrm{GHz}$ opacity of $0.08-0.1$. Data were reduced in the STARLINK package, using the same procedures and calibrators outlined in section 2.3 of Tetarenko et al. (2017). JCMT flux densities of the source in both epochs are reported in Table B2. We note that we only detect the source at $350 \mathrm{GHz}$ in these epochs; however, $3 \sigma$ upper limits in the $666 \mathrm{GHz}$ band are provided in the table. Given the rapidly changing (sub)-mm flux density observed in this outburst, we also attempted to create maps of the source on shorter time-scales (less than the full observation period). To do this, we used a custom procedure we developed to produce a data cube, containing multiple maps of the target source region, at different time intervals throughout our observation (see section 3.2 of Tetarenko et al. 2017 for the details of this procedure). In both epochs, we were only able to measure the flux density on time-scales as short as the 30 min scan time-scale, as V404 Cyg was too faint, and the noise was too high to accurately measure the flux density on shorter time-scales.

\subsubsection{December 2015/January 2016 mini-outburst}

As V404 Cyg displayed renewed activity in December 2015/January 2016, we also observed V404 Cyg with the JCMT (project code M15BI036) on 2016 January 1 and 2 (MJDs 57388 and 57389). Each observation consisted of one $\sim 30$ min scan on target with the SCUBA-2 detector (using the daisy configuration), from 19:48 to 20:20 UTC (MJD 57388.825 to 57388.847) on January 1 and from 19:59 to 20:32 UTC (MJD 57389.833 to 57389.856 ) on January 2. CRL2688 was used for absolute flux calibration on January 1, and Mars was used on January 2. During both observations, we were in the Grade 1 weather band, with a $225 \mathrm{GHz}$ opacity of $0.04 / 0.05$ on January $1 / 2$. These later epochs were also reduced in the STARLINK package, following the same procedures as in Section 2.5. V404 Cyg transits during the daytime at this time of year; therefore, the JCMT was operating in an specialized extended observing mode at the time of our observations. As we observed the (sub)-mm flux density change on rapid time-scales during the main outburst of V404 Cyg, we opted to also search for variability within the January 1 observation by splitting the scan into two maps. The first half of the observation shows an average flux density of $58 \pm 19 \mathrm{mJy}$ and the second half shows an average flux density of $38 \pm 10 \mathrm{mJy}$. The source was not bright enough, and the noise was too high to accurately measure the flux density on shorter time-scales. For the same reasons, we were unable to obtain flux density measurements on time-scales less than the $30 \mathrm{~min}$ scan time-scale in the January 2 epoch. JCMT flux densities of the source in these later epochs are also reported in Table B2.

\subsection{IR/optical/UV/X-ray observations}

We have compiled publicly available OIR, UV, and X-ray (Chan$d r a$, Plotkin et al. 2017a; Swift/XRT, Sivakoff et al. 2015b) photometric observations that were quasi-simultaneous with our radio through sub-mm observations (i.e. $<1 \mathrm{~d}$ separation from our observations). Observational details and flux densities from these data are reported in Table B3, where data in this table have been dereddened (when required) using the prescription in Cardelli, Clayton \& Mathis (1989), with an $E(B-V)=1.3 \pm 0.2$ (Casares et al. 1993). Additionally, in our analysis we include time-resolved OIR data from Kimura et al. (2016) and AAVSO, ${ }^{5}$ as well as INTEGRAL X-ray data (3-10 and 60-200 keV bands from the JEM-X and ISGRI instruments, respectively; Rodriguez et al. 2015), ${ }^{6}$ all occurring simultaneously with our radio/sub-mm data sets.

The Swift/XRT data were all taken in photon counting mode, and we analysed the data using the HEASOFT software package. We first reprocessed the data using xrtpipeline, and then we extracted source and background spectra using standard procedures in xselect. Due to the presence of dust scattering haloes around V404 Cyg in many of these observations (e.g. Beardmore et al. 2016; Heinz et al. 2016), background spectra were extracted from regions where no haloes were detected. We fit the $0.5-10 \mathrm{keV} X$-ray spectra with an absorbed power law (TBABS*PEGPWRLW); here, we used abundances from Wilms, Allen \& McCray (2000) and cross-sections from Verner et al. (1996). We tied the power-law photon index ( $\Gamma=$ $1.8 \pm 0.3 ; 90$ per cent confidence interval) together, but allowed the hydrogen absorption column to vary between observations $\left(N_{\mathrm{H}} \sim\right.$ $\left.0.5-3 \times 10^{22} \mathrm{~cm}^{-2}\right)$. We report the flux densities arising from these fits at $5 \mathrm{keV}\left(1.21 \times 10^{9} \mathrm{GHz}\right)$.

\section{RESULTS}

\subsection{Light curves}

Daily time-scale light curves of all of our radio through sub-mm observations of V404 Cyg are presented in the top two panels of Fig. $1 .^{7}$ Throughout our month long-monitoring of the source, we

\footnotetext{
${ }^{5}$ Observations from the AAVSO International Database (Kafka 2018), https: //www.aavso.org.

${ }^{6}$ All INTEGRAL X-ray data presented in this paper are taken from the INTEGRAL public data products available at http://www.isdc.unige.ch/ in tegral/analysis\#QLAsources (Kuulkers 2015; PI: Rodriguez).

${ }^{7}$ All the fluxes presented in this figure are measured through imaging the source over the full observation period, except for the AMI $16 \mathrm{GHz}$ data, where a weighted mean of time-resolved measurements (100 s time-bins) is taken.
} 


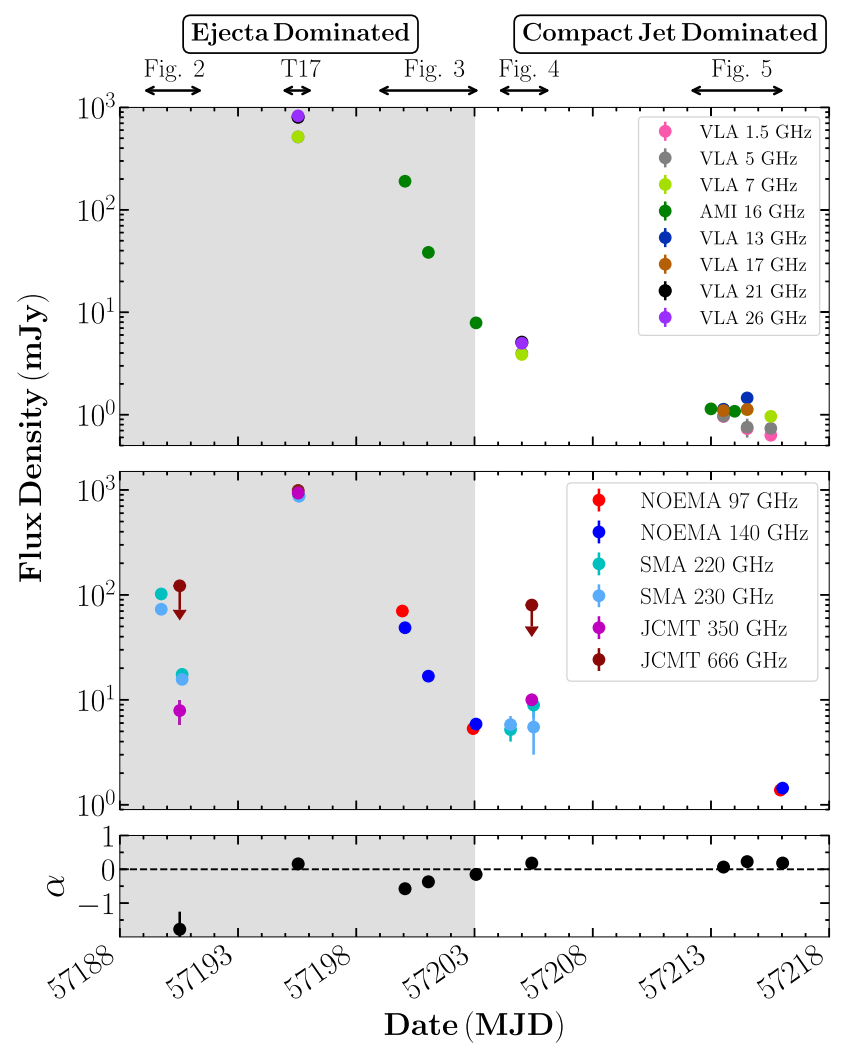

Figure 1. Day-time-scale light curves of V404 Cyg during its 2015 June outburst. The top panel displays the radio frequency bands, the middle panel displays the (sub)-mm frequency bands, and the bottom panel displays the radio-sub-mm spectral indices, in epochs where at least two different bands were sampled (using the convention $f_{v} \propto v^{\alpha}$, where $\alpha$ represents the spectral index; dotted line indicates $\alpha=0$ ). The (un-)shaded regions represent timeperiods where the jet emission was likely dominated by jet ejecta or a compact jet (as labelled above the top panel). The figures displaying timeresolved measurements of these data are indicated at the top of the figure (T17 indicates Tetarenko et al. 2017). Over our month long-monitoring period of V404 Cyg, we find that the jet emission is highly variable, where the radio through sub-mm fluxes vary by around three orders of magnitude, and the spectral index varies between steep $(\alpha<0)$ and inverted $(\alpha>0)$.

observe the radio/sub-mm flux to vary by over three orders of magnitude, ranging from Jy levels at its brightest to sub-mJy levels at its faintest. In the sub-mm bands, the emission in our first epoch (taken hours after the first detection of the outburst in X-rays) is relatively bright compared to the $\mathrm{mm} / \mathrm{sub}-\mathrm{mm}$ flux densities typically seen in BHXBs (i.e. $\sim 100 \mathrm{mJy}$ versus $<50 \mathrm{mJy}$ ), but rapidly drops by an order of magnitude within the next $24 \mathrm{~h}$. Following our first two detections, the sub-mm flux likely continues to rise, approaching a peak on MJD 57195. The source then begins to decay, where this decay is initially quite rapid (i.e. the flux density drops at least an order of magnitude between MJD 57195 and 57199), before the emission appears to plateau for a few days around MJD 57204, and then proceeds to decay at a much slower rate as the source heads towards quiescence. The radio emission tracks the sub-mm emission closely, and both show a potential secondary peak in the light curves around MJD 57200.

The bottom panel of Fig. 1 displays the radio through sub-mm spectral indices (where a single power law is fit across radio/sub$\mathrm{mm}$ frequencies), for epochs where at least two different bands were sampled. We find that the spectral indices appear to vary between
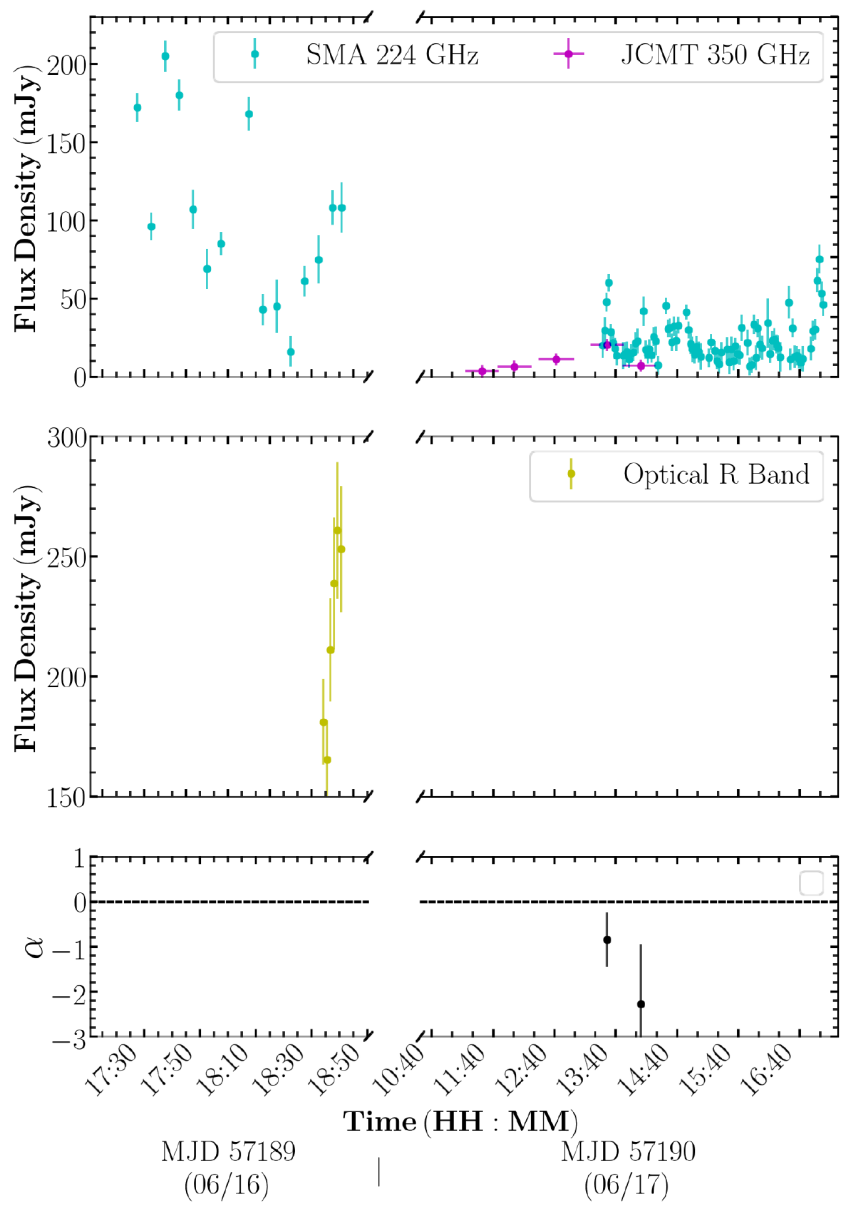

Figure 2. Time-resolved light curves and spectral indices of V404 Cyg during the first two days of our monitoring of the outburst (June 16 and 17, MJDs 57189 and 57190). The data shown have varying time-bin sizes; $224 \mathrm{GHz}$ (5/2 min June 16/17), $350 \mathrm{GHz}$ (30 min), Optical $R$ band (75 s; Kimura et al. 2016). Here, we have combined the two SMA sidebands (cyan data points) in order to gain a higher signal to noise in our time-resolved light curves. The horizontal error bars on the JCMT measurements (magenta data points) represent the time range of the $30 \mathrm{~min}$ SCUBA-2 scans. The dotted line in the bottom panel indicates a spectral index of $\alpha=0$. In less than $24 \mathrm{~h}$, between these two epochs, both the sub-mm flux levels and variability amplitude change dramatically.

steep $(\alpha<0)$ and inverted $(\alpha \geq 0)$ during the MJD 57189-57204 period, but then remain flat to inverted for the rest of our monitoring period.

As V404 Cyg is known to be significantly variable regardless of its brightness, these day-time-scale light curves and spectral indices will only display the overall average trend in the data. Therefore, we opted to also search for intra-observation variability in our data. To do this, we created time-resolved light curves (and simultaneous spectral index measurements) of all of our radio through sub-mm observations. These time-resolved light curves, along with simultaneous optical and X-ray data (when available), are displayed in Figs 2-5. To ensure that any short time-scale variations we observe from V404 Cyg are dominated by intrinsic variations, and not atmospheric or instrumental effects, we extracted high time resolution measurements from our calibrator sources as well. We find that the majority of our calibrator observations show relatively constant fluxes (variations $<10$ per cent of the average flux density), except for the SMA data taken on MJD 57189 (see discussion below). 


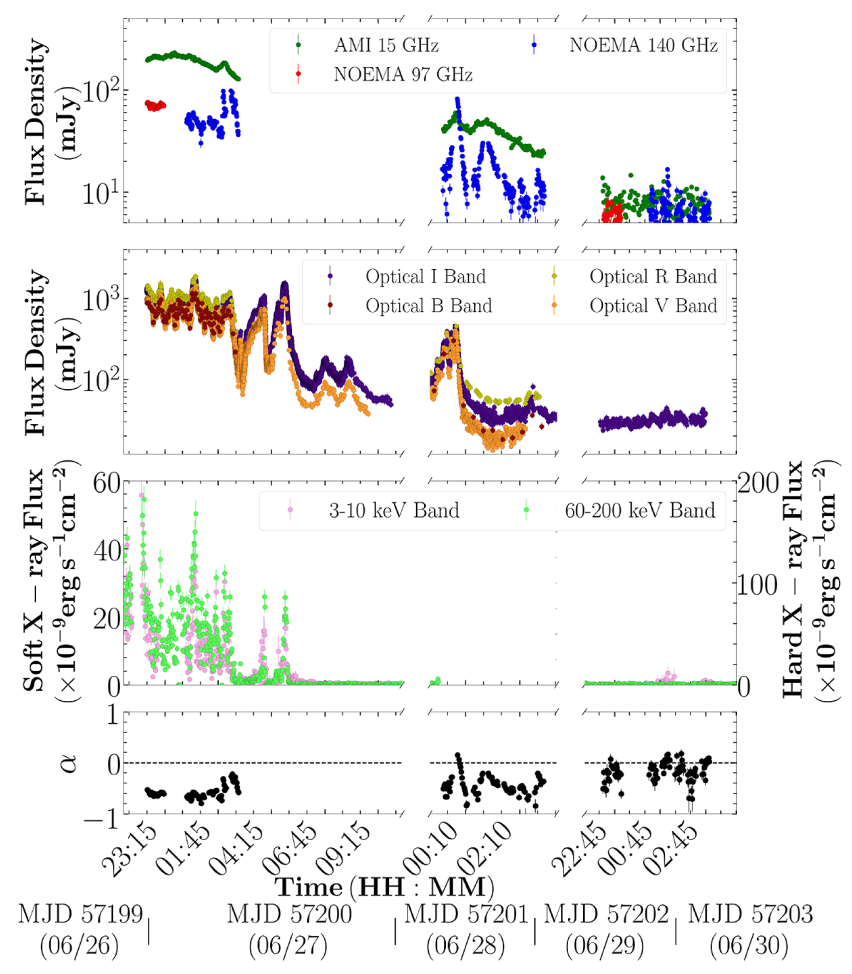

Figure 3. Time-resolved light curves and spectral indices of V404 Cyg between June 26 and 30 (MJD 57199-57203). The data shown have varying time-bin sizes: $15 \mathrm{GHz}(100 \mathrm{~s}), 97-140 \mathrm{GHz}(45 \mathrm{~s})$, optical $I, R, B, V$ bands (75 s; Kimura et al. 2016), INTEGRAL 3-10 keV (64 s), INTEGRAL 60$200 \mathrm{keV}$ (64 s). The dotted line in the bottom panel indicates a spectral index of $\alpha=0$. We initially detect rapid flaring activity at radio through $\mathrm{X}$-ray frequencies, which drops in amplitude, becomes less frequent, and eventually stops all together, over this four day period.

To characterize the amplitude of any intra-observation variability and compare between epochs, we use the fractional RMS statistic,

$F_{\mathrm{var}}=\sqrt{\frac{S^{2}-\bar{\sigma}_{\mathrm{err}}^{2}}{\bar{x}^{2}}}$

where $\bar{x}$ represents the weighted mean of the flux measurements, the sample variance $S^{2}=\frac{1}{N-1} \sum_{i=1}^{N}\left(x_{i}-\bar{x}\right)^{2}$, and the mean square measurement error $\bar{\sigma}_{\text {err }}^{2}=\frac{1}{N} \sum_{i=1}^{N} \sigma_{\text {err }, i}^{2}$ (Akritas \& Bershady 1996; Vaughan et al. 2003; Sadler et al. 2006). For this paper, we consider $F_{\text {var }}<20$ per cent as not significantly variable, 20 per cent $<F_{\text {var }}<$ 50 percent as mildly variable, and $F_{\text {var }}>50$ percent as highly variable.

In our first epoch on MJD 57189, the sub-mm emission is highly variable over the short $\sim 1.5 \mathrm{~h}$ observation, with a $F_{\text {var }}=71.0 \pm$ 1.4 per cent at $224 \mathrm{GHz}$. Although, we note that this variability appears to be very stochastic (especially when compared to the next epoch taken $\sim 24 \mathrm{~h}$ later), rather than showing smooth or structured variations that we might expect to see from this high-frequency emission probing close to the jet base (e.g. see Tetarenko et al. 2017). Upon examining the calibrator light curve for this observation, we noticed that the calibrator source shows an atypically high level of variability within the first half of the observation (i.e. prior to $\sim 18: 00$ UT), likely due in large part to the very low elevations of these observations (as low as $15 \mathrm{deg}$. Therefore, all of the V404 Cyg variability observed in this epoch may not be intrinsic to the source. Less than a day later, the variability amplitude decreases, along with the average flux level, to just above mildly variable at
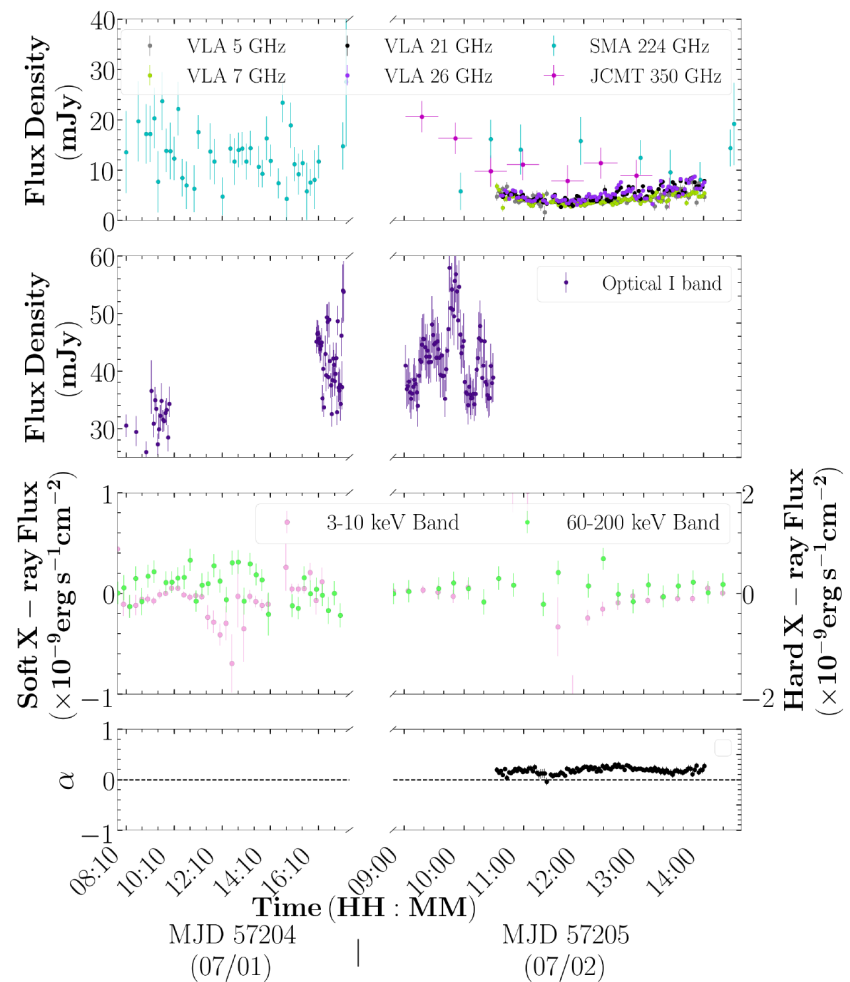

Figure 4. Time-resolved light curves and spectral indices of V404 Cyg on July 01 and 02 (MJDs 57204 and 57205). The data shown have varying timebin sizes: $5-26 \mathrm{GHz}(2 \mathrm{~min}), 224 \mathrm{GHz}$ (10/30 min on July 1/2), $350 \mathrm{GHz}$ (30 min), optical $I$ band (100/75 s on July 01/02; AAVSO/ Kimura et al. 2016), INTEGRAL 3-10 keV (15 min), INTEGRAL 60-200 keV (15 min). Here, we have combined the two SMA sidebands in order to reduce the noise in our short time-scale light curves. The horizontal error bars on the JCMT measurements represent the range of the $30 \mathrm{~min}$ SCUBA- 2 scans. The dotted line in the bottom panel indicates a spectral index of $\alpha=0$. While no structured flaring activity is observed, the sub-mm emission remains highly variable on July 01 , before becoming much more stable a day later.

$F_{\text {var }}=51.9 \pm 0.8$ per cent at $224 \mathrm{GHz}$. While we are only able to sample the sub-mm spectral index in two time bins on MJD 57190, both show a steep spectral index in this epoch, consistent with that observed from the daily average data (see Figs 1 and 2).

Our next epoch, taken approximately a week later (MJD 57195), displayed large scale, structured flaring activity, with lower frequency emission appearing as a smoothed and delayed version of high-frequency emission. We have shown that this emission can be well modelled by a series of bi-polar, adiabatically expanding jet ejections (details of this data set are reported in Tetarenko et al. 2017). This structured flaring activity (tracing repeated jet ejection events) likely continued intermittently up to MJD 57199, where we sample a final, large radio flare (peaking at $\sim 200 \mathrm{mJy}$ at $16 \mathrm{GHz}$ ), coinciding with rapid flaring activity at optical and $\mathrm{X}$-ray frequencies (see Fig. 3). Following this large flare, the radio through optical emission remains highly variable for another $1-2 \mathrm{~d}$ $\left(F_{\text {var }}=71.6 \pm 0.2\right.$ per cent at $140 \mathrm{GHz}$ on MJD 57200), displaying multiple smaller amplitude flaring events, before the flaring activity ceases, and the variability amplitude drops to mildly variable $\left(F_{\text {var }}=39.0 \pm 0.2\right.$ per cent at $140 \mathrm{GHz}$ on MJD 57202/57203). The spectral indices during the flaring activity between MJD5719957201 oscillate between steep and inverted on hourly time-scales, consistent with the evolving optical depth of adiabatically expand- 


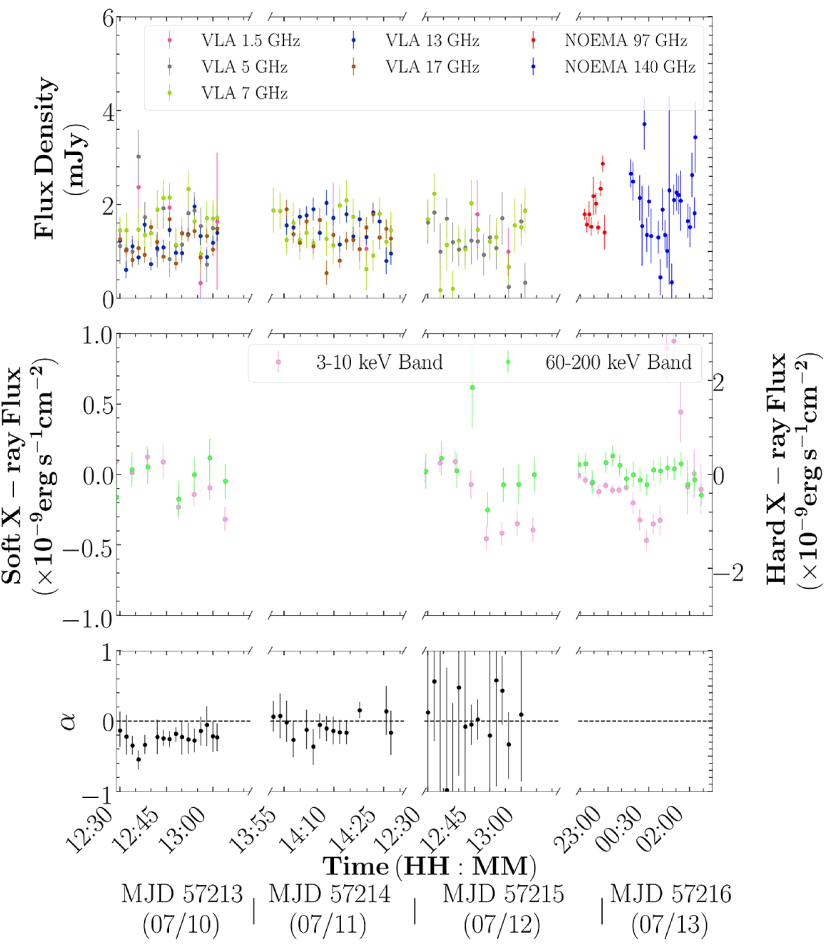

Figure 5. Time-resolved light curves and spectral indices of V404 Cyg between July 10 and 13 (MJD 57213-57216). The data shown have varying time-bin sizes; $1.5 \mathrm{GHz}$ (10 $\mathrm{min}), 5-17 \mathrm{GHz}$ (2 $\mathrm{min}), 97-140 \mathrm{GHz}$ (5 min), INTEGRAL 3-10 keV (5/15 min on MJD 57213-57215/57216), INTEGRAL 60-200 keV (5/15 min on MJD 57213-57215/57216). The dotted line in the bottom panel indicates a spectral index of $\alpha=0$. The radio through sub-mm flux densities are much more constant in these epochs, when compared to our observations one week earlier (see Fig. 4).

ing jet ejecta. After the flaring activity had ceased, the spectral index is much more stable over time, and close to flat.

In the following $2 \mathrm{~d}$, the sub-mm variability amplitude once again increases to highly variable with $F_{\text {var }}=85 \pm 5$ per cent at $224 \mathrm{GHz}$ on MJD 57204, before declining to the point where any variance in the data is much less than the measurement errors on MJD 57205 (see Fig. 4). The optical and X-ray emissions at this time are not significantly variable $\left(F_{\mathrm{var}}<10\right.$ per cent), while the spectral index is stable, and remains inverted across radio through sub-mm bands. A week later (MJD 57213), both the radio and submm flux has dropped by another order of magnitude, while the X-ray flux has remained relatively constant (close to zero) since MJD 57201 (see Fig. 5). All the radio bands are not significantly variable, displaying $F_{\text {var }}<20$ per cent (similar to that observed in later radio observations taken in late July and early August; Plotkin et al. 2017a), and the sub-mm variability amplitude is similar to that seen on MJD 57202/57203. The spectral indices remain flat to slightly inverted during the MJD 57213-57216 period.

\subsection{Modelling the flaring activity}

Out of all the time-resolved light curves shown in Figs 2-5, the radio and sub-mm light curves between MJD 57199 and 57201 show a distinct morphology. During these days we observe rapid, multifrequency flaring activity (with the lower frequency emission appearing to be a smoothed, delayed version of the higher frequency emission; see Fig. 3). Given the striking similarity between these data and our earlier work on multifrequency flaring activity from MJD 57195 (Tetarenko et al. 2017), we opted to apply the jet model we developed for that data set to the MJD 57199-57201 light curves.

While a detailed description of our jet model is provided in section 4.2 of Tetarenko et al. (2017), we provide a brief summary here. Our V404 Cyg jet model reproduces emission from multiple, discrete, ballistically moving jet ejection events on top of a constant compact jet component with a power-law spectrum. Each of the ejection events consists of the simultaneous launching of identical bi-polar plasma clouds, both of which evolve under the van der Laan (van der Laan 1966) synchrotron bubble formalism. Additionally, our model folds in both projection and relativistic effects (e.g. relativistic beaming and geometric time delays) for each ejection event.

Here, we use the same modelling process detailed in section 4.3 of Tetarenko et al. (2017), where we implement a Markov chain Monte Carlo (MCMC) algorithm to fit our light curves on MJD $57200 / 57201$ (Foreman-Mackey et al. 2013). The best-fitting parameters and their uncertainties ${ }^{8}$ for this fit are shown in Table 1 , and Fig. 6 displays the best-fitting model overlaid on our light curves (see also Fig. C1). With our best-fitting model, we find that a total of 5 bi-polar ejection events can reproduce the overall morphology and flux densities of the emission we observe in the June 27/28 (MJD 57200/57201) epoch. Further, our modelling suggests that the inclination angle of the jet axis changes by up to $\sim 40$ deg during this series of ejections, which is consistent with the magnitude of jet axis precession independently estimated from a series of resolved jet ejecta observed in an earlier epoch with the VLBA (Miller-Jones et al. in preparation). Therefore, overall our modelling shows that the flaring emission observed on June 27/28 (MJD 57200/57201) is consistent with emission originating from multiple, discrete jet ejection events. While we also observe multifrequency flaring in the 2015 June 26/27 (MJD 57199/57200) epoch, there is limited overlap between the $\mathrm{mm} / \mathrm{sub}-\mathrm{mm}$ and radio observations, which makes it difficult to reliably fit this data set with our model.

We note that it is possible that the best-fitting parameters presented in Table 1 do not represent a completely unique solution due to degeneracies in our model (where different combinations of parameters can reproduce similar flaring profiles). Further, the modelling presented in this paper is not as well constrained as our previous application of the model to the flaring from an earlier epoch (MJD 57195; Tetarenko et al. 2017), as we only have light curves at two simultaneous bands (as opposed to 8 simultaneous bands on MJD 57195) to constrain the model. We caution that the reader should keep this caveat in mind in the further discussion of jet properties in this paper.

\subsection{Cross-correlation analysis}

To search for time lags between different frequency bands, we computed cross-correlation functions (CCFs) of our time-resolved light curves in all epochs, using the $z$-transformed discrete correlation function (ZDCF; Alexander 1997, 2013). We chose to use

\footnotetext{
${ }^{8}$ The uncertainties reported in Table 1 are purely statistical, only representing confidence intervals on our parameters under the assumption that our model completely represents the data. However, given the residuals with respect to our best-fitting model, it is possible that there are physical/instrumental effects in the data that cannot be reproduced by our model. See section 4.3 of Tetarenko et al. (2017) for a more detailed discussion on this point.
} 
Table 1. V404 Cyg jet model best-fitting parameters.

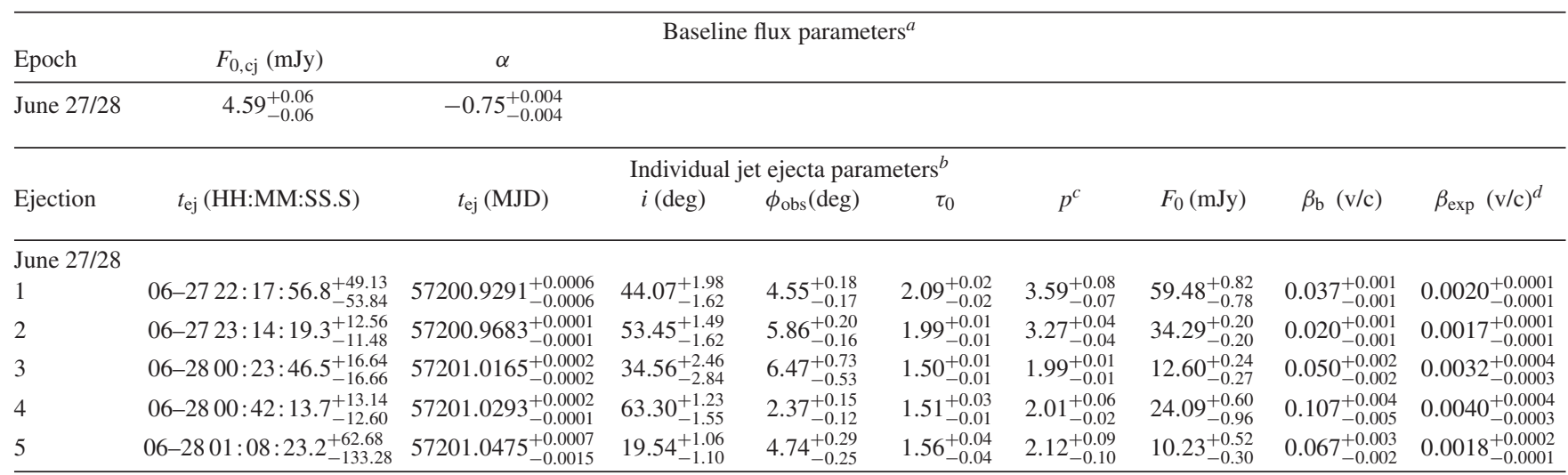

Notes. ${ }^{a}$ The baseline flux (representing contributions from a compact jet) is best fit by a single power law, with amplitude $F_{0, \mathrm{cj}}$ at $140 \mathrm{GHz}$, and spectral index $\alpha$.

${ }^{b}$ Ejecta parameters are defined as follows: ejection time $\left(t_{\mathrm{ej}}\right)$, inclination angle of the jet axis $(i)$, jet opening angle $\left(\phi_{\mathrm{obs}}\right)$, synchrotron optical depth (at $\left.140 \mathrm{GHz}\right)$ at the time of peak flux density $\left(\tau_{0}\right)$, peak flux density of the ejecta component $\left(F_{0}\right)$, and the bulk ejecta speed $\left(\beta_{b}\right)$.

${ }^{c}$ The index of the electron energy distribution, $p$, is not a fitted parameter but rather is solved for using values of $\tau_{0}$. Similar to the situation discussed in our earlier modelling work (Tetarenko et al. 2017), the large range of energy indices $(p)$ for the ejecta found here is not entirely physical for a single source. We believe that more extreme values of the energy index could be mimicking the effect of physics that has not been included in our model.

${ }^{d}$ The expansion velocity, $\beta_{\text {exp }}$, is not a fitted parameter but rather is solved for using values of $\beta_{\mathrm{b}}, i, \phi_{\text {obs }}$.

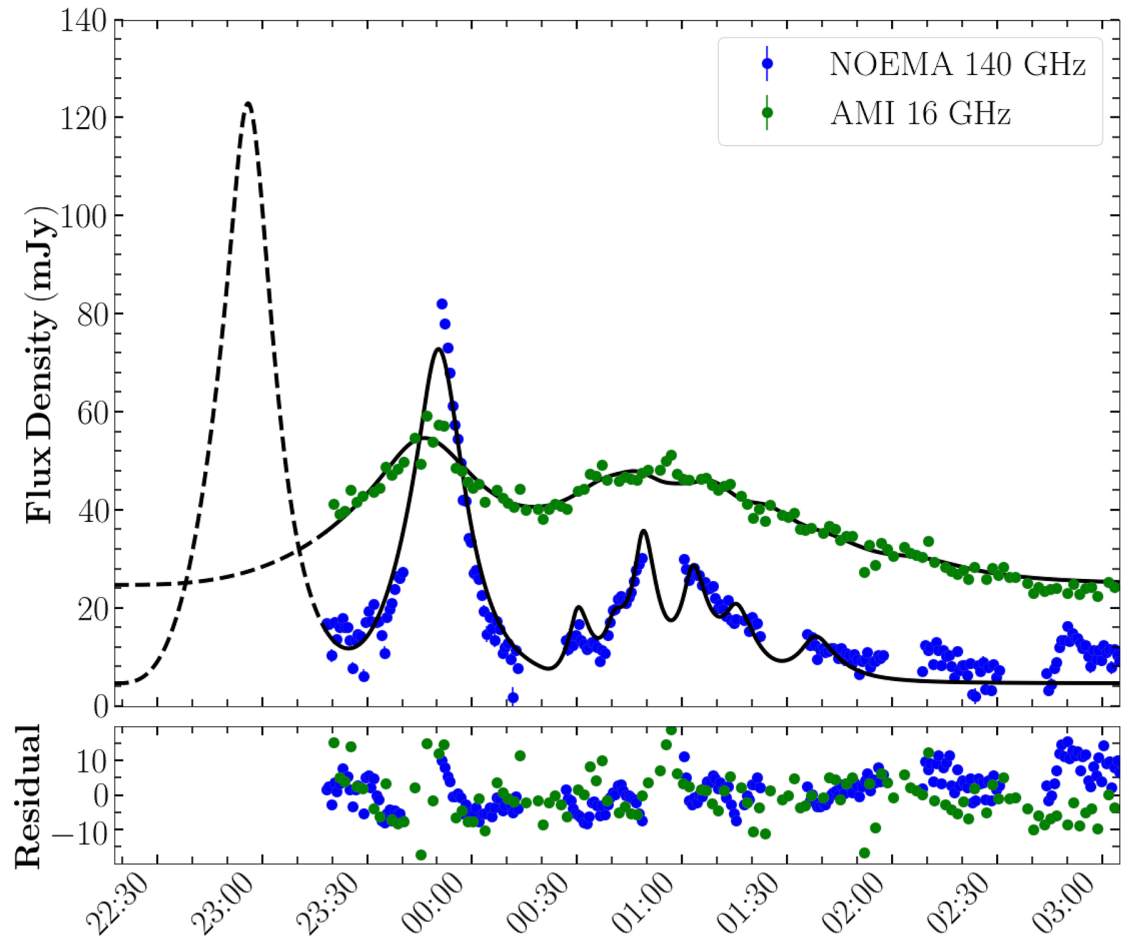

Time on 2015 June $27 / 28$ (HH : MM)

Figure 6. Radio (AMI $16 \mathrm{GHz}$ ) and mm/sub-mm (NOEMA $140 \mathrm{GHz}$ ) light curves of V404 Cyg on 2015 June 27/28 (MJD 57200/57201). In the top panel, we have overlaid our predicted best-fitting jet model at each frequency on the light curves (black lines, where our model contains contributions from both approaching and receding components for each ejection event). The residuals are shown in the bottom panel, where residual $=($ data-model $) /($ observational errors). Our best-fitting model, which contains a total of 5 bi-polar ejection events, can reproduce the overall morphology and flux levels of the emission we observe from V404 Cyg, indicating that this flaring emission is consistent with emission originating from multiple, discrete jet ejection events. Note that we do not attempt to model all of the peaks and wiggles in the $\mathrm{mm} / \mathrm{sub}-\mathrm{mm}$ emission past 02:00 UT, as we do not have the radio frequency coverage to constrain any additional components in the model past this point. See Fig. $\mathrm{C} 1$ for a version of this figure where we decompose the full model into individual approaching and receding components. 
the $\mathrm{ZDCF}$ algorithm, as this method has been shown to provide a more robust estimate of the CCFs for sparse, unevenly sampled light curves, when compared to the classic discrete correlation function (Edelson \& Krolik 1988) or the interpolation method (Gaskell \& Peterson 1987). To obtain an estimate of the CCF peak (indicating the strongest positive correlation, and thus the best estimate of any time-lag between the light curves from different frequency bands), with corresponding uncertainties, we utilize the maximum likelihood method ${ }^{9}$ described in Alexander (2013). Additionally, to estimate the significance level of any peak in the $\mathrm{CCF}$, we perform a set of simulations. For these simulations, we randomize each radio light curve 1000 times (i.e. Fourier transform the light curves, randomize the phases, then inverse Fourier transform back, to create simulated light curves that share the same power spectra as the real light curves), and calculate the CCF for each randomized case. We then determine the fraction of simulated CCF data points above the peak CCF level in the original CCF run. Performing these simulations allows us to quantify the probability of false detections in our CCFs, by accounting for stochastic fluctuations and intrinsic, uncorrelated variability within each radio light curve.

While we performed the above CCF analysis for all of the radio through X-ray data sets for which we had overlapping, time-resolved data, we only find clear evidence of time lags during the July 02 (MJD 57205) epoch (see Fig. 7). In particular, we measure a time lag between the $26 \mathrm{GHz}$ radio band and the $5 \mathrm{GHz}$ radio band of $12.0_{-4.2}^{+3.7} \mathrm{~min}$. However, the measured time lags between the $26 \mathrm{GHz}$ band and the $7 / 21 \mathrm{GHz}$ bands are consistent with a zero lag within the uncertainty limits (where the $1 \sigma$ upper limits for the $26 \mathrm{GHz}$ to $7 / 21 \mathrm{GHz}$ lags are $<10 \mathrm{~min}$ and $<5 \mathrm{~min}$, respectively). Further, the optical $I$-band flaring activity observed in this epoch (see Fig. 4) is unlikely to be correlated with this radio emission, as a simple jet model $\left(z_{0} \propto 1 / v\right.$, where $z_{0}$ represents the distance down the jet axis from the black hole) paired with our detected radio lag predicts a $\sim 15$ min lag between the $I$ band and $5 \mathrm{GHz}$, rather than the hours between the optical and radio flaring observed in the light curves (see Figs 4 and 7). However, as the radio and $I$-band light curves do not overlap in this epoch, we cannot rule out a correlation between the two. Therefore, we are unable to conclusively determine if a trend with frequency, where the lower frequency bands always lag the higher frequency bands (and the lag increases as the frequency decreases in the comparison band), exists in our CCFs. Such a trend is expected from emission originating in a compact jet, as lower frequency emission is expected to originate from a region further down the jet axis from the black hole, and these time lags between radio bands could trace the propagation of material downstream along the jet (Malzac et al. 2003; Casella et al. 2010; Gandhi et al. 2017; Vincentelli et al. 2018).

As the jet model, we used in Section 3.2 can predict lags between different frequency bands for each jet ejection event, it is of interest to compare the model predicted lags from data on MJD 57200/57201 and the CCF predicted lags from data on MJD 57205. In particular, our best-fitting jet model from MJD 57200/57201

\footnotetext{
${ }^{9}$ This method estimates a fiducial interval rather than the traditional confidence interval. The approach taken here is similar to Bayesian statistics, where the normalized likelihood function (fiducial distribution) is interpreted as expressing the degree of belief in the estimated parameter, and the 68 percent interval around the likelihood function's maximum represents the fiducial interval ( 68 per cent of the likelihood-weighted ensemble of all possible CCFs reach their peaks within this interval).
}

predicts time lags between 26 and $5 \mathrm{GHz}$ of 24-59 min, between 26 and $7 \mathrm{GHz}$ of $17-44 \mathrm{~min}$, and between 26 and $21 \mathrm{GHz}$ of 2-5 min, for different ejection events. Therefore, the jet model predicted lags between 26 and $5 \mathrm{GHz}$ are all larger than our $\mathrm{CCF}$ measured lag. These differing lags likely indicate varying jet properties at different phases of the outburst (i.e. decline from a major flare versus flat-spectrum compact jet emission at a much lower level), such as bulk speed, inclination angle, opening angle, or electron energy distributions, between the MJD 57200 and 57205 epochs.

\subsection{Broad-band spectra}

In the epochs following the structured flaring activity (MJD 57203 57216), where the emission is much more constant (showing minimal flux variability within an observation), we constructed broadband spectra to track the spectral evolution of the jet emission as V404 Cyg decayed towards quiescence (including additional radio data from the Giant Metrewave Radio Telescope reported in Chandra \& Kanekar 2017, to add lower frequency coverage at $0.235,0.610$, and $1.280 \mathrm{GHz}$ ). We fit these radio through optical/UV broad-band spectra with a phenomenological multicomponent model, consisting of a broken power law (representing compact jet emission), a blackbody $\left(R_{\star}=5.71 \mathrm{R}_{\odot}, T_{\star}=0.784 \mathrm{~T}_{\odot}\right.$, representing the known companion star; Gallo et al. 2007), and in two epochs, an additional single power law (representing emission from fading jet ejecta). To fit these spectra, we use an MCMC algorithm (Foreman-Mackey et al. 2013), where the best-fitting result is taken as the median of the one-dimensional posterior distributions, and the uncertainties are reported as the range between the median and the 15 th percentile $(-)$, and the 85 th percentile and the median $(+)$, corresponding approximately to $1 \sigma$ errors. These broad-band spectra are displayed in Fig. 8, and the best-fitting model parameters are reported in Table 2 . We note that while accretion disc emission has been known to contribute to the optical/UV emission in broad-band spectra of BHXBs (Khargharia, Froning \& Robinson 2010 estimate $<3$ per cent accretion disc contamination during quiescence for V404 Cyg, although the accretion disc is much brighter in outburst than in quiescence; Bernardini et al. 2016), we do not include an accretion disc component in our model presented here (e.g. Hynes et al. 2002; Hynes 2005). While we could reasonably reproduce the optical/UV emission in our broad-band spectra with a cool $(T$ $\sim 3000 \mathrm{~K}$ ), highly truncated $\left(R \sim 10^{4} R_{g}\right.$ ), viscous disc (where irradiation is not necessary to describe the spectral shape), the integrated flux over this disc emission implies a physically improbable mass transfer rate (e.g. on MJD $57205 \sim 2 \times 10^{-9} \mathrm{M}_{\odot} \mathrm{yr}^{-1}$ ) through the disc for this scenario. Therefore, we favour a model where the jet dominates the optical/UV emission in our broad-band spectra.

The broad-band spectra constructed from data on MJD 5720257205 are well fit by a broken power law, where we detect the spectral break in the near-IR bands. The OIR emission from the companion star is fainter than the jet emission on MJDs 57204 and 57205, contributing little to the overall broad-band spectra (although the jet and companion star show similar flux levels in the OIR on MJD 57202/57203). However, on MJDs 57202/57203, and 57204 , we require an additional power-law component to account for the excess emission at radio frequencies. ${ }^{10}$ The broken power-

\footnotetext{
${ }^{10}$ Note that we have fixed the spectral index of this jet ejecta component to a typical value of -0.7 in the MJD 57202/57203 epoch, as we only have a
} 

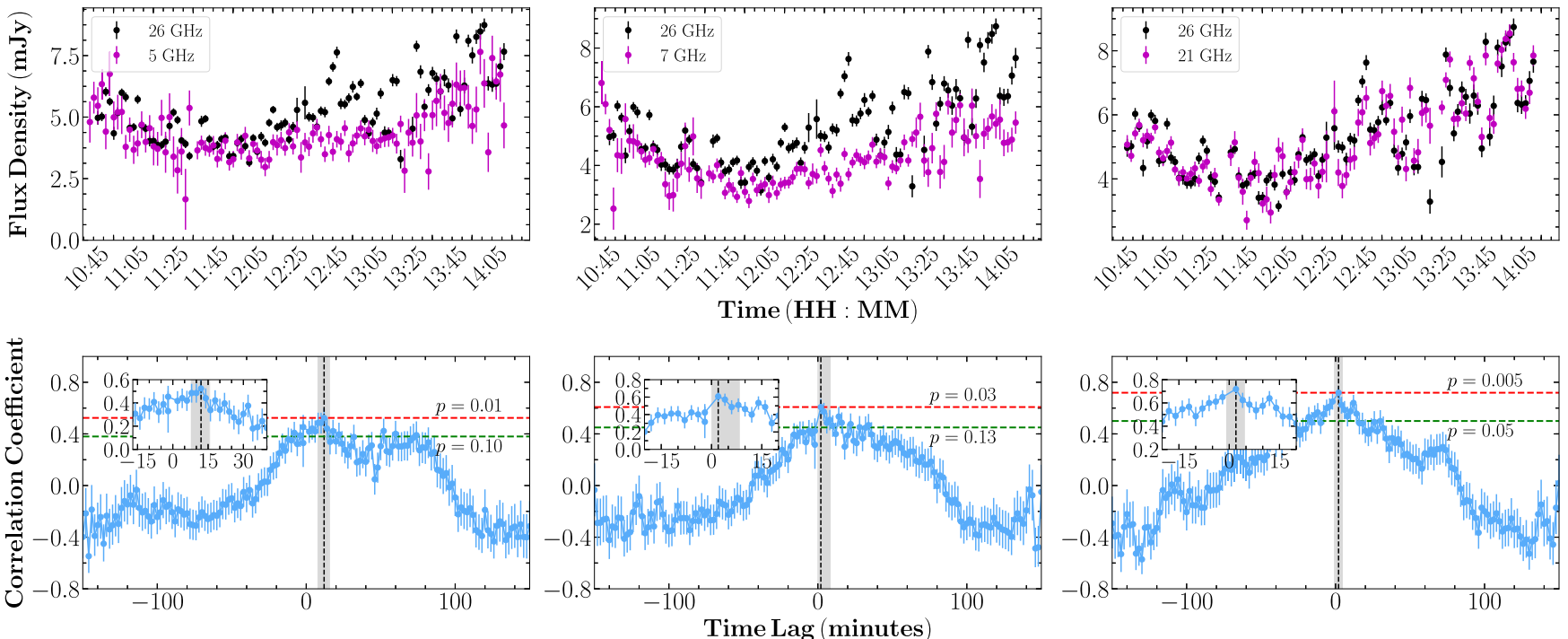

Figure 7. Radio light curves (zoomed in versions of Fig. 4; top panels) and CCFs (bottom panels) between the VLA radio frequency bands on July 02 (MJD 57205). The peak of each CCF (indicating the strongest positive correlation) is shown by the black dotted line, with the 68 per cent fiducial confidence interval indicated by the shaded grey region, and different significance levels indicated by the red and green dotted lines (see Section 3.3 for details). The insets in each panel display a zoomed view of the region surrounding the peak of each CCF. A positive time lag indicates that the lower frequency band lags behind the higher frequency band. We observe a clear time lag between the 26 and $5 \mathrm{GHz}$ bands (bottom left; $12.0_{-4.2}^{+3.7}$ min); however, our measured lags between the $26 \mathrm{GHz}$ and the $7 \mathrm{GHz}$ (bottom middle)/21 GHz (bottom right) bands are consistent with zero lag.

law emission is characteristic of a compact jet, while the additional power-law component could originate in emission from fading jet ejecta, potentially launched during the flaring period $2-3 \mathrm{~d}$ prior to these epochs. Further, while we see very little evolution in the location of the spectral break across these three broad-band spectra, the optically thin spectral index may steepen over time, while the optically thick spectral index may flatten over time (although, given the large uncertainties in some epochs, it is difficult to determine if we see an evolutionary trend in this spectral index; e.g. MJD 57204, where we only sample the optically thick part of the spectrum in two closely placed sub-mm bands).

The broad-band spectra constructed from data on MJD 5721357216 are also well fit by a broken power law, where we detect the spectral break at frequencies as high as the optical bands. This indicates that the spectral break has moved to higher frequencies over the $\sim 1$ week time-scale between these epochs and the previous three epochs. Further, during these later epochs, the jet emission has faded by $\sim$ an order of magnitude, and thus the emission from the companion star contributes much more to the overall spectral shape in these broad-band spectra.

We note that all of the above conclusions are dependent upon the assumption that other emission sources (e.g. accretion disc emission, irradiation of the companion star by X-rays) are not significantly contaminating the OIR/UV bands in our broad-band spectra. For instance, X-rays emitted during the final major flaring event of the outburst (occurring a few days prior to the spectra presented in this work) could have potentially caused the emission from the companion star to be much brighter than normal (through the irradiation process), and in turn contribute more to the OIR/UV part of the broad-band spectrum. In this case, we would expect

single radio data point to constrain this component. However, as we have three radio data points in the MJD 57204 epoch, we allow this parameter to vary in the fit for that epoch. a smaller jet contribution to the OIR/UV emission. In fact, this scenario may explain the larger deviations between the radio/sub$\mathrm{mm}$ data and our best-fitting model on MJD 57213. The presence of a hotter companion star component, producing more OIR/UV flux, would allow for a flatter jet spectrum at lower frequencies, which would be more representative of the radio/sub-mm data in this epoch.

Further, given the high spectral break frequency measured here, it is of interest to explore whether the synchrotron jet emission could be dominating the emission in the X-ray bands during our sampled epochs. To test this scenario, we have also included the available quasi-simultaneous Swift XRT/Chandra X-ray flux measurements (in the $0.5-10 \mathrm{keV}$ band) within our broad-band spectra (Fig. 8 and Table B3). Simply extrapolating the optically thin part of the jet spectrum to the X-ray bands in the X-ray sampled epochs (blue dotted lines on June 29/30 and July 02, and grey shading representing a range of expected optically thin spectral indexes between $\alpha=-0.6$ and $\alpha=-0.8$ on July 10 and 11) clearly overpredicts the X-ray flux. Therefore, we consider the possibility where a second break, representing a synchrotron cooling break ${ }^{11}$ (due to the highest-energy electrons losing their energy through radiation on time-scales faster than the dynamical time-scale; Sari, Piran \& Narayan 1998; Pe'er \& Markoff 2012; Russell et al. 2013a, 2014), occurs between the UV and X-ray bands in the synchrotron spectrum. To place constraints on the location of the cooling break in this case, we consider the July 02 (MJD 57205) epoch, as this is the only epoch in which we have data sampling the optically thin part of the jet spectrum, no contribution from jet ejecta, and an X-ray measurement. Through refitting the July 02 spectrum, including the X-ray data point, adding

\footnotetext{
${ }^{11}$ We note that to the best of our knowledge the synchrotron cooling break has only been detected in the broad-band spectra of one BHXB so far (MAXI J1836-194), where the cooling break was found in the optical bands between $(3-4.5) \times 10^{14} \mathrm{~Hz}$ (Russell et al. 2014).
} 

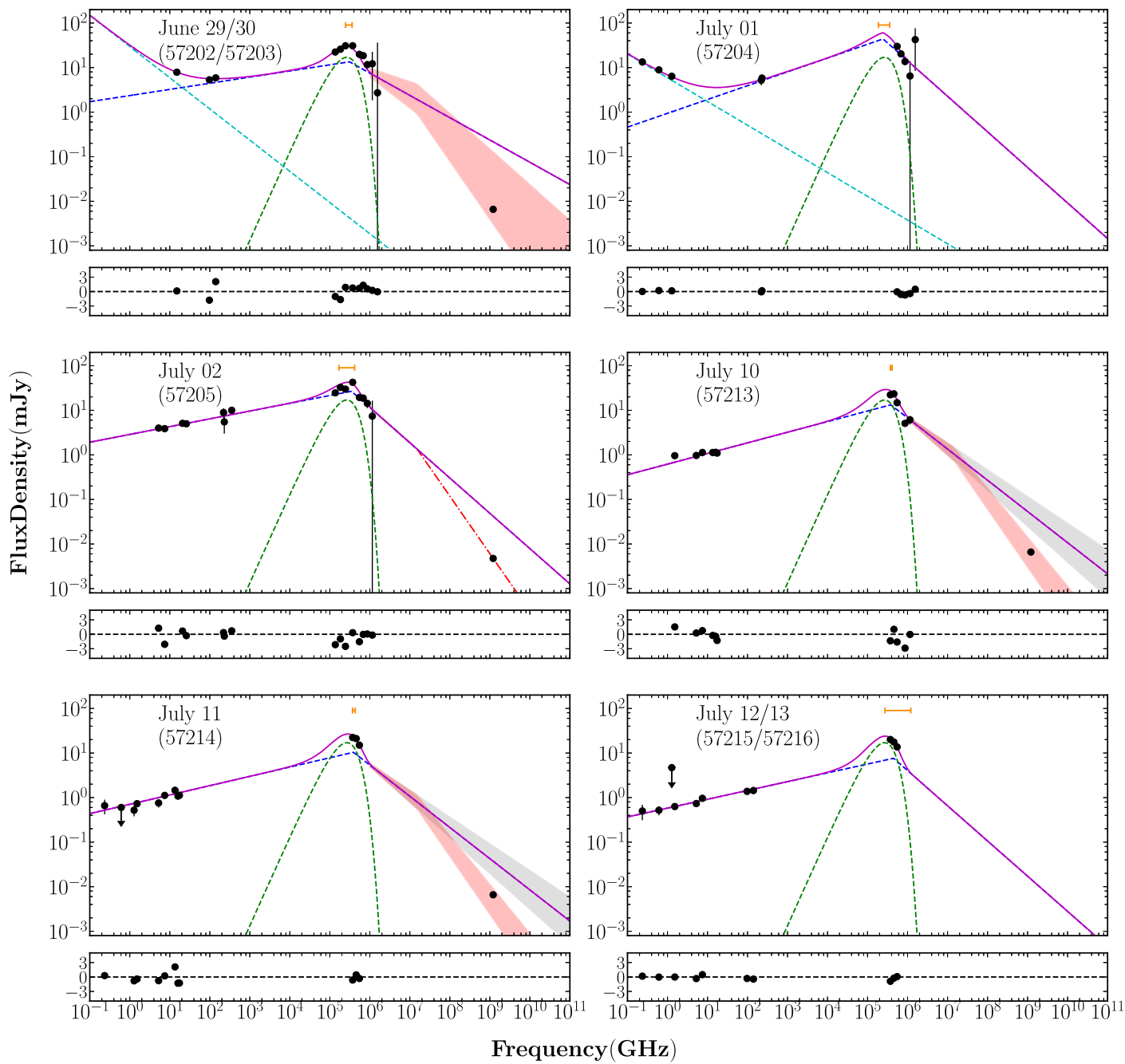

Figure 8. Broad-band spectra of V404 Cyg during the decay of the 2015 outburst. The top panel in each broad-band spectrum displays the broad-band photometric data (black markers), and the best-fitting model (where the Swift XRT/Chandra X-ray data points are not included in these fits) in each epoch. The solid purple line represents the total model, and the dotted lines represent the model components (green is the companion star component, dark blue is the compact jet component, and cyan is the fading jet ejecta component). The orange bars/arrows represent constraints on the location of the spectral break The dash-dotted red line and shaded red/grey regions represent constraints on the synchrotron jet contribution to the X-ray emission (where our jet model overpredicts X-ray flux unless a sychrotron cooling break is considered in the spectrum; see Section 3.4 for details). The bottom panel of each broad-band spectra represents the residuals, where residual $=($ data model $) /($ observational errors $)$. The emission in these broad-band spectra is consistent with being dominated by emission from a compact jet, with a spectral break in the near-IR and optical frequency bands.

Table 2. Best-fitting parameters for broad-band spectral modelling ${ }^{a, b}$.

\begin{tabular}{|c|c|c|c|c|c|c|c|}
\hline Date & $\nu_{\text {break }}(\mathrm{GHz})$ & $F_{\text {break }}(\mathrm{mJy})$ & $\alpha_{\text {thick }}$ & $\alpha_{\text {thin }}$ & $L_{\text {jet }}\left(\mathrm{erg} \mathrm{s}^{-1}\right)$ & $F_{15 \mathrm{GHz}}(\mathrm{mJy})$ & $\alpha_{\text {thin }, 2}$ \\
\hline June 29/30 & $\left(3.10_{-0.63}^{+0.50}\right) \times 10^{5}$ & $13.48_{-0.90}^{+0.53}$ & $0.14_{-0.01}^{+0.01}$ & $-0.50_{-0.29}^{+0.22}$ & $\left(5.47_{-0.97}^{+1.14}\right) \times 10^{35}$ & $4.45_{-0.32}^{+0.25}$ & -0.70 \\
\hline July 01 & $\left(2.44_{-0.57}^{+1.12}\right) \times 10^{5}$ & $43.97_{-0.29}^{+0.50}$ & $0.31_{-0.03}^{+0.11}$ & $-0.80_{-0.10}^{+0.18}$ & $\left(1.42_{-0.28}^{+0.33}\right) \times 10^{36}$ & $1.33_{-0.69}^{+0.56}$ & $-0.53_{-0.16}^{+0.11}$ \\
\hline July 02 & $\left(3.48_{-1.79}^{+0.69}\right) \times 10^{5}$ & $26.71_{-4.14}^{+2.10}$ & $0.17_{-0.01}^{+0.02}$ & $-0.79_{-0.18}^{+0.37}$ & $\left(9.45_{-2.91}^{+2.81}\right) \times 10^{35}$ & - & - \\
\hline July 10 & $\left(3.81_{-0.31}^{+0.06}\right) \times 10^{5}$ & $13.27_{-1.56}^{+0.92}$ & $0.24_{-0.01}^{+0.01}$ & -0.70 & $\left(5.25_{-0.81}^{+0.92}\right) \times 10^{35}$ & - & - \\
\hline July 11 & $\left(3.89_{-0.20}^{+0.44}\right) \times 10^{5}$ & $10.38_{-1.64}^{+1.59}$ & $0.20_{-0.02}^{+0.02}$ & -0.70 & $\left(4.22_{-0.79}^{+1.10}\right) \times 10^{35}$ & - & - \\
\hline July $12 / 13$ & $\left(4.48_{-1.75}^{+7.58}\right) \times 10^{5}$ & $7.62_{-2.67}^{+1.21}$ & $0.19_{-0.02}^{+0.04}$ & -0.70 & $\left(3.03_{-1.05}^{+1.19}\right) \times 10^{35}$ & - & - \\
\hline
\end{tabular}

Notes. ${ }^{a}$ Columns from left to right: spectral break frequency, flux at the spectral break, optically thick spectral index, optically thin spectral index, integrated compact jet power $\left(L_{\text {jet }}=4 \pi D^{2} \int \nu L_{v} \mathrm{~d} \nu\right.$, from 1.5 to $\left.1.2 \times 10^{6} \mathrm{GHz}\right)$ given our best-fitting model, flux (at $\left.15 \mathrm{GHz}\right)$ and spectral index of additional optically thin power-law component. The radius and temperature of the companion star were fixed in all fits $\left(R_{\star}=5.71 \mathrm{R}_{\odot}, T_{\star}=0.784 \mathrm{~T} \odot\right.$; Gallo et al. 2007).

${ }^{b}$ Note that we fix the optically thin spectral index to a value of -0.7 for the July 10 , July 11 , and July $12 / 13$ epochs, as we do not have sufficient data to place accurate constraints on this parameter in our fitting. 
a cooling break (where the spectral index after the cooling break is steeper by $\Delta \alpha=0.5$; Sari et al. 1998; Russell et al. 2014) in the model, and keeping all other parameters fixed at the original bestfit values, we find $v_{\text {coolbr }}=\left(1.5_{-0.5}^{+0.7}\right) \times 10^{16} \mathrm{~Hz}$ (where the cooling break version of the model is displayed as a red dot-dashed line in the middle-left panel of Fig. 8). Given this cooling break measurement, and estimates of the optically thin spectral index (we assume spectral indexes between $\alpha=-0.6$ and $\alpha=-0.8$ in epochs where we have no constraint on this parameter), we also extrapolate the synchrotron spectrum to the X-ray bands in the June 29/30, as well as the July 10 and 11 epochs (displayed as red shading in the top left-hand, middle right-hand, and bottom left-hand panels of Fig. 8). Overall, we find that the jet synchrotron emission could reasonably be producing a significant fraction of the X-ray flux in these epochs. Further discussion of the plausibility of this scenario is presented in Section 4.2.

\section{DISCUSSION}

Throughout the June 2015 outburst of V404 Cyg, the jet emission we observe displays a wide range of intensities (spanning over three orders of magnitude between the brightest and faintest epochs), and the spectral and variability properties of the jet emission change dramatically throughout the outburst (on time-scales of minutes to days). In this work, we have presented detailed diagnostics of this jet emission, and in the following sections, we discuss jet properties and evolution in V404 Cyg, as well as draw comparisons to the jet emission observed in the 1989 outburst, and the December 2015/January 2016 mini-outburst.

\subsection{Jet ejecta behaviour}

For the first $\sim 13 \mathrm{~d}$ of the June 2015 outburst, the jet emission from V404 Cyg appears to be dominated by emission from discrete jet ejections, as evidenced by the structured multifrequency flaring activity in the light curves, and the rapidly oscillating radio through sub-mm spectral indices (consistent with the evolving optical depth of these expanding ejecta; see Figs 1 and 3). In recent work (Tetarenko et al. 2017), we developed a jet ejecta model for V404 Cyg that could reproduce the brightest multifrequency flaring emission detected during the outburst (on MJD 57195), and in turn allow us to probe jet speeds, energetics, and geometry. To examine how the jet ejecta properties could evolve throughout the outburst, in this paper we have presented model fits to another multifrequency flaring data set, occurring $5 \mathrm{~d}$ following the brightest epoch. We find that these later multifrequency flaring episodes can also be well represented by emission from a series of jet ejections (Fig. 6). Upon comparing the jet ejecta properties between our modelled data sets, the later epochs tend to show fainter ejecta (tens to hundreds of $\mathrm{mJy}$, rather than thousands of mJy), with lower bulk speeds $(<0.1 c$ versus $0.2-0.6 c$ on MJD 57195; Tetarenko et al. 2017), and longer periods between ejections (on the order of hours, rather than minutes). This suggests that the ejecta properties changed throughout the flaring period, becoming slower, less energetic, and less frequent as the outburst progressed, before the discrete jet ejections stopped all together.

If the jet ejecta launched from V404 Cyg are powered by the accretion flow, then the radio/sub-mm emission we observe should be correlated with the optical/X-ray emission (where the optical emission could originate in the jet base/acceleration region and/or be reprocessed X-ray emission from the accretion flow; e.g. Gandhi et al. 2017). In particular, we might expect ${ }^{12}$ to observe optical/Xray flaring counterparts preceding our radio/sub-mm flares, where the ejection times predicted by our modelling should coincide with the optical/X-ray flaring. Interestingly, the beginning of the optical flaring complex observed on June 27 at $\sim 23: 00$ UT coincides with the predicted ejection time for the largest $\mathrm{mm} / \mathrm{sub}-\mathrm{mm}$ flare (ejection 2 in Table 1, also see Fig. C2) observed in this epoch. Additionally, while we were unable to model the radio/sub-mm flaring on June 26 , if we assume similar delay time-scales $(\sim 10-40 \mathrm{~min}$ between $\mathrm{mm} / \mathrm{sub}-\mathrm{mm}$ and optical/X-ray) from our modelled epoch, it seems plausible that the rapid optical/X-ray activity on June 26 could also be related to the radio/sub-mm flaring we observe during this epoch (see Fig. 3). However, not all of the ejection events we model on June 27 have clear optical counterparts (e.g. ejections 3-5; see Fig. C2). The explanation for the lack of clear optical/Xray counterparts to some radio/sub-mm flares in this system is not known, but additional factors such as a precessing accretion disc (Miller-Jones et al. in preparation) obscuring the jet base or emission being absorbed by the strong accretion disc wind detected from the inner accretion disc in this source (Munoz-Darias et al. 2016) could be affecting the optical/X-ray emission we observe, thus making it more difficult to identify counterparts to the radio/sub-mm flares. Therefore, our observations provide hints of a possible correlation between radio/sub-mm and optical/X-ray emission during jet ejection events in V404 Cyg, but we do not fully understand the connection between the accretion flow and jet emission in this system.

\subsection{Compact jet behaviour}

Following the flaring period, the jet emission from V404 Cyg switches to being dominated by a compact jet, as evidenced by the flat/inverted (radio through sub-mm) spectral indices, and the absence of large-scale flaring activity in the time-resolved light curves in all bands (see Figs 4 and 5).

Through applying a phenomenological model to the broad-band emission at this stage in the outburst, we find that the compact jet is the dominant source of emission in V404 Cyg from the radio through optical/UV bands (see Fig. 8, where the broad-band spectra can be well fit by a broken power law, characteristic of compact jet emission). With this modelling, we initially detect the optically thick to thin synchrotron jet spectral break in the near-IR bands $\left(\sim 2-3 \times 10^{14} \mathrm{~Hz}\right)$, and find that the spectral break could reach as high as the optical bands in our final epochs $\left(4.48 \times 10^{14} \mathrm{~Hz}\right)$. This high spectral break frequency is atypical for BHXBs (typical values for the spectral break in BHXBs are $10^{11-14} \mathrm{~Hz}$; Russell et al. 2013a), but consistent with data from the 1989 outburst, which also display a high spectral break frequency $\left(\sim 1.8 \times 10^{14} \mathrm{~Hz}\right.$; Russell et al. 2013a). These results are also in agreement with the recent work of Maitra et al. (2017), who present several lines of evidence to suggest that the optical emission was dominated by the compact jet on MJD 57200, and theorize the spectral break lies above the optical $V$ band at this time $\left(\sim 5.5 \times 10^{14} \mathrm{~Hz}\right)$. As the spectral break

\footnotetext{
${ }^{12}$ We note that while multiband flaring activity detected in some epochs may appear to be occurring simultaneously, observing no delay between $\mathrm{X}$-ray/optical/sub-mm/radio emission does not make physical sense in the accretion flow/jet picture. Emission from the X-ray/optical/sub-mm/radio bands are emitted from different regions that are expected to be separated by measurable light traveltimes. As such, any appearance of simultaneity is more likely to be a sampling effect in our light curves.
} 
probes the jet base (where jet particles are first accelerated to high energies), Maitra et al. (2017) theorize that a spectral break at such a high frequency suggests the jet base was very compact and energetic at this point in the outburst. If this is the case, our spectral break measurements imply these conditions persisted as the system faded towards quiescence.

Measuring a spectral break in the optical bands in the V404 Cyg jet spectrum could also have important implications regarding the jet contribution to the X-ray emission in this system. In particular, with such a high spectral break frequency, the optically thin synchrotron emission from the jet could be dominating the emission in the X-ray bands (e.g. in XTE J1550-564 the jet has been shown to dominate the X-ray bands during the outburst decay; Russell et al. 2010). In Section 3.4 and Fig. 8, we have shown that a synchrotron spectrum (with a cooling break between the UV and X-ray bands) extrapolated to the $\mathrm{X}$-ray bands can reasonably reproduce the $\mathrm{X}$-ray fluxes observed during the decay of this V404 Cyg outburst. This indicates that the jet could be producing a large fraction of the X-ray flux at this point in the outburst. However, to confirm this theory, a more detailed X-ray analysis (possibly examining hard/soft lags, reflection features, or the presence of iron lines) would need to be performed to verify that the X-ray emission is indeed more consistent with synchrotron from a jet rather than Comptonization in a hot inner flow. Such an X-ray analysis is beyond the scope of this work. However, we note that X-ray spectral studies (Motta et al. 2017b) of this stage of the outburst find photon indexes $(\Gamma \sim 1.5-1.7)$ consistent with our estimated optically thin synchrotron spectral indices ( $\alpha \sim-0.6$ to -0.8 , where $\Gamma=1-\alpha$ ). This suggests that the $\mathrm{X}$ ray spectrum may show a similar slope to the optically thin part of the compact jet spectrum, and thus be indicative of a synchrotron origin for this X-ray emission.

Further, we see limited evidence for evolution in the broad-band spectra across our sampled epochs during the outburst decay. For instance, the optically thin spectral index stays relatively constant (within error) across the epochs where it is measured, the optically thick spectral index may only flatten slightly over time, and the spectral break resides in the near-IR/optical bands across all of our sampled epochs (see Table 2). Additional radio observations occurring after our sampled epochs (in late July and early August) also show a similar trend, where the shape of the radio spectrum (i.e. spectral index) remains relatively constant over time (Plotkin et al. 2017a). Limited jet spectral evolution may suggest that the jet properties are not changing significantly as the jet emission fades during these epochs (e.g. the jet spectral shape can be sensitive to many parameters, such as the magnetic field strength at the base of the jet, jet geometry, inclination of the system, the particle acceleration process, and the electron energy distribution injected into the jets; Kaiser 2006; Pe'er \& Casella 2009; Jamil, Fender \& Kaiser 2010; Malzac 2014). Interestingly, Chandra \& Kanekar (2017) have reported the detection of a spectral break in the radio band at $v_{\text {break }}=$ $1.8 \mathrm{GHz}$ on MJD 57199/57200. This finding is consistent with our previous work (Tetarenko et al. 2017), where we found evidence of a compact jet with a spectral break between $0.341<v_{\text {break }}<5.25 \mathrm{GHz}$ on MJD 57195. Therefore, while we observe little spectral break evolution in broad-band spectra sampling epochs later than MJD 57202 in this work, combining these two results may suggest that the spectral break rapidly shifted from the radio to near-IR bands over the span of a few days (between MJD 57199-57202). Moreover, we find that the compact jet emission during the outburst decay can also be highly variable (on minute to hour time-scales), similar to the conditions observed in the quiescent V404 Cyg jet. While the variability amplitude at radio/sub-mm frequencies appears to follow an average trend, where the variability amplitude decreases as the intensity of the jet emission decreases, interestingly we also find that the variability amplitude can sporadically increase in certain time periods. For example, on MJD 57204 the sub-mm emission displays a large variability amplitude of $F_{\text {var }} \sim 85$ per cent, while the epochs taken $\sim 24$ hours prior to and following the MJD 57204 epoch, show little to no variability (see Fig. 4). Maitra et al. (2017) have suggested that strong optical frequency variability (also probing the jet base region, but closer to the black hole than probed by sub-mm frequencies) occurring a few days earlier on MJD 57200, could be caused by a disruption in the feeding of the jet. In this situation, the mass outflow rate changes sporadically in response to a change in the mass inflow rate through the disc. Alternatively, the high variability amplitude detected on MJD 57204 could be tracing the re-establishment of the compact jet, following the last major flaring episodes occurring a few days earlier. In either case, if the jet flow was unstable in the MJD 57204 epoch, this could also explain the origin of the time lags we detect between the radio bands in the MJD 57205 epoch. In particular, these time lags could be tracking a disturbance in the jet flow, which was injected into the jet base sometime between the MJD 57204/57205 epochs, and has since propagated downstream (to lower frequencies) in the jet.

\subsection{Comparison to the 1989 outburst}

V404 Cyg underwent three known outbursts prior to 2015: 1938 (when the optical counterpart was observed and originally identified as Nova Cygni; Wagner et al. 1989), 1956 (as discovered on photographic plates; Richter 1989), and 1989 (when the transient X-ray counterpart was first identified by the Ginga satellite; Makino 1989). The 1989 outburst was monitored at multiple frequencies, including X-ray, optical, and radio frequencies (Han \& Hjellming 1992). While both the 1989 and 2015 outbursts have been shown to exhibit similar X-ray behaviour (e.g. bright X-ray flaring activity that is not always intrinsic to the source), a detailed comparison between the radio jet behaviour during these different outbursts has not yet been presented. Radio frequencies offer the distinct advantage of providing a cleaner view of the system, when compared to the $\mathrm{X}$-ray regime, as the high column density/external obscuration effects (Motta et al. 2017a) do not apply in the radio regime. As such, given our well-sampled radio coverage, in this section we compare the radio jet behaviour between the 1989 and 2015 outbursts (see Fig. 9 for the radio frequency light curves and radio spectral indices for both outbursts).

The radio jet behaviour in the 1989 and 2015 outbursts display many similarities; both outbursts reach similar peak intensities $(\sim 1 \mathrm{Jy})$, the spectral indices show similar evolution (progresses from steep, to flat, to inverted, in $\sim 20 \mathrm{~d}$ ), both outbursts spend $\sim 15 \mathrm{~d}$ in a high luminosity flaring accretion state before transitioning into a hard accretion state (where the jet emission is dominated by a compact jet; red and blue shading in Fig. 9), the radio emission remained variable throughout the outburst (regardless of flux level), and there are hints of coupling between radio, optical, and X-ray emissions (Han \& Hjellming 1992; Plotkin et al. 2017a; Tetarenko et al. 2017). However, the radio emission in the 2015 outburst decays significantly faster than the radio emission in the 1989 outburst $(\sim 30 \mathrm{~d}$ in 2015 versus $\sim 300 \mathrm{~d}$ in 1989 , to reach submJy levels), and no mini-outburst is observed at radio frequencies in 1989 (Muñoz-Darias et al. 2017). However, given the duration of the 2015 mini-outburst, and the sampling time-scale between the 1989 radio epochs, it is entirely possible that such a mini-outburst 


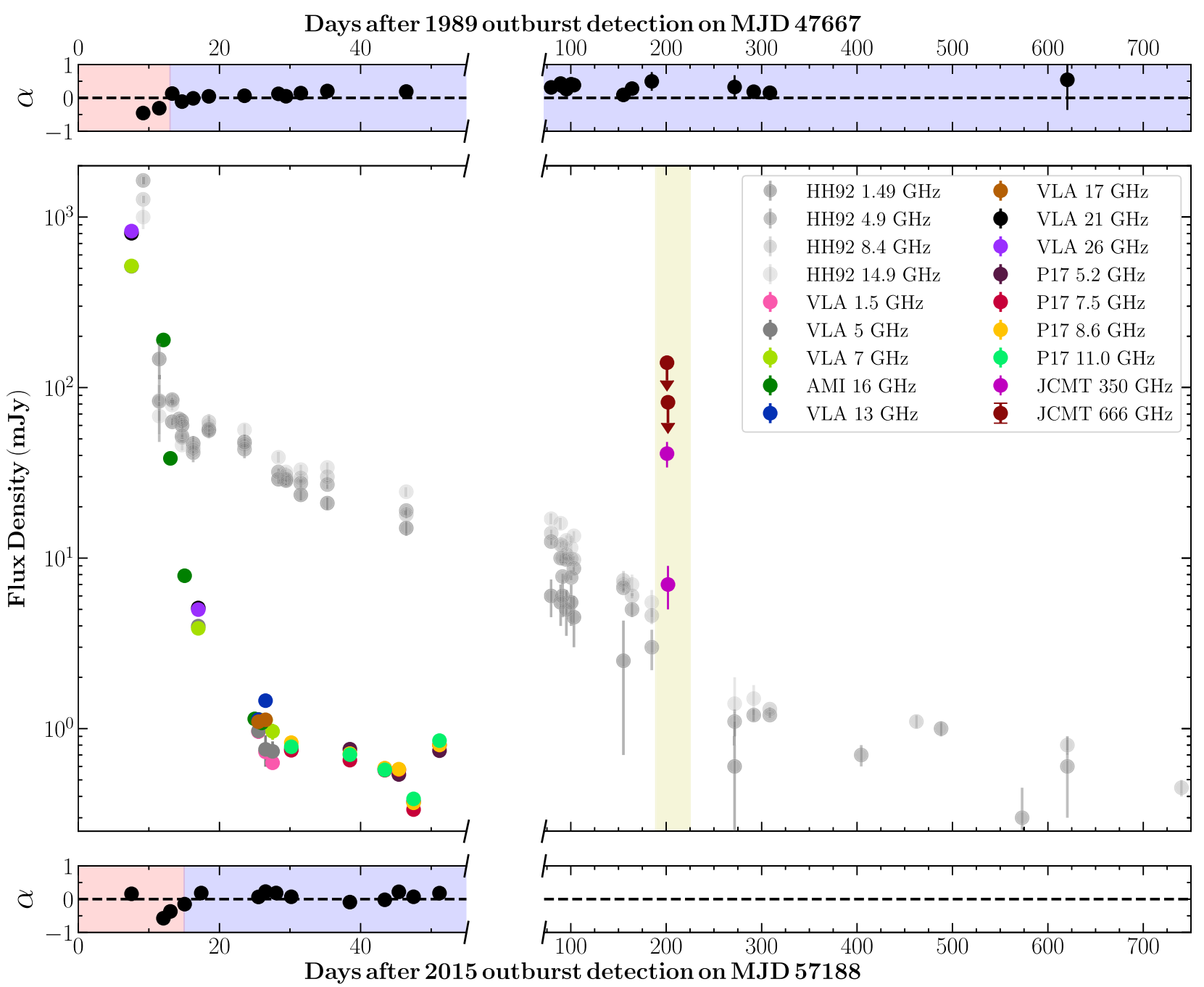

Figure 9. Radio frequency light curves of the 1989 (grey-scale data points) and 2015 (coloured data points) outbursts of V404 Cyg. The 1989 radio frequency data are taken from Han \& Hjellming 1992 (HH92), and we supplement our 2015 radio frequency coverage with the measurements reported in Plotkin et al. 2017a (P17). The top and bottom panels indicate the radio spectral indices (in epochs where at least two radio bands were observed) in 1989 and 2015, respectively. The shaded regions in the top and bottom panels represent the time periods in which the radio emission was likely dominated by jet ejecta emission (red; $\alpha<0$ ) or compact jet emission (blue; $\alpha \geq 0$ ). We also include our JCMT sub-mm coverage of the 2015 mini-outburst in this plot, where the yellow shading in the middle panel indicates the duration of the 2015 mini-outburst period (MJD 57377-57413; Muñoz-Darias et al. 2017). While both outbursts reach similar peak intensities and show similar radio spectral index evolution, the radio emission from the 2015 outburst appears to decay much faster than in the 1989 outburst.

was simply missed in 1989. Interestingly, the 2015 outburst also decayed much more quickly in X-rays when compared to the 1989 outburst ( $\sim 60$ days in 2015 versus $\sim 160 \mathrm{~d}$ in 1989; Terada et al. 1994; Oosterbroek et al. 1997; Zycki, Done \& Smith 1999a; Plotkin et al. 2017a).

Several works (Gallo, Fender \& Pooley 2003b; Corbel et al. 2008; Gallo et al. 2014; Plotkin et al. 2017a; Gallo, Degenaar \& van den Eijnden 2018) have shown that the radio luminosity of V404 Cyg is linked to the X-ray luminosity through a robust discjet coupling relationship (radio luminosity is proportional to the X-ray luminosity; $L_{R} \propto L_{X}^{\beta}$, where $\beta \sim 0.54$ for V404 Cyg). This relationship was shown to hold across a wide range of $X$-ray luminosities $\left(L_{X} \sim 10^{32}-10^{37} \mathrm{erg} \mathrm{s}^{-1}\right)$ and is valid for both the 1989 and 2015 outbursts (Plotkin et al. 2017a). Therefore, as the X-ray luminosity can be thought of a proxy for mass accretion rate, the more rapid drop in radio luminosity in the 2015 outburst could be the result of a more rapid drop in the average mass accretion rate after the peak of the outburst, when compared to the 1989 outburst. Munoz-Darias et al. (2016) have suggested that the strong accretion disc wind detected during the 2015 outburst may be a factor that regulates outburst duration, as these winds can significantly deplete the mass in the accretion disc (and potentially cause drastic and rapid changes in mass accretion rate). As such, it is possible that the faster decay seen in our radio light curves in the 2015 outburst indicates that the mass loss rate in the winds was much higher in the 2015 outburst, leaving less matter in the disc to fuel the jets. Along the same lines, the length of the quiescent period prior to each outburst may also be a contributing factor to the more rapid radio decay. In particular, as the quiescent period prior to the 1989 outburst was longer than the quiescent period prior to the 2015 outburst ( 33 versus $26 \mathrm{yr}$ ), the system had more time to build up mass in the disc (to fuel the jets) before the 1989 outburst. 


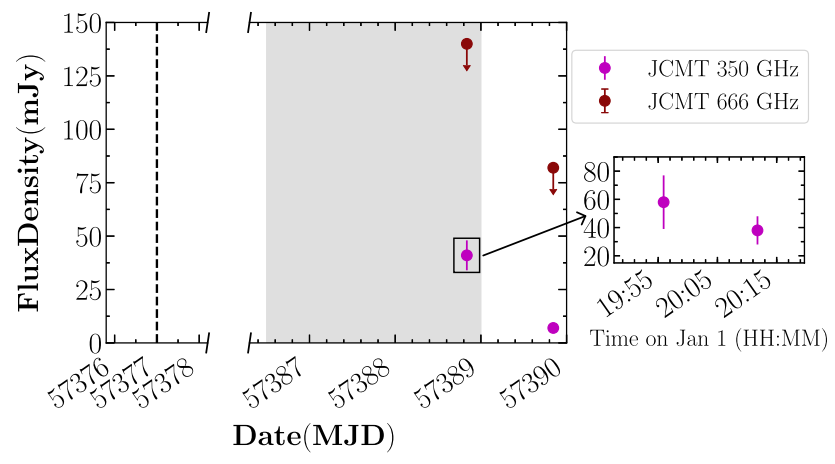

Figure 10. JCMT sub-mm light curves of V404 Cyg during the December 2015 mini-outburst. The vertical dotted line indicates the start of the minioutburst on MJD 57377, while the shaded grey region indicates the most active flaring phase of the mini-outburst (MJD 57386.5-57389, as identified by Muñoz-Darias et al. 2017). The right-hand panel displays a time-resolved analysis of the 2016 January 1 (MJD 57388) observation, where we split the 30 min scan into two segments. The decreasing flux trend observed in the daily light curve (main panel) and time-resolved measurements (right-hand panel), as well as the timing of our measurements near the end of the active flaring period, suggests that we may have caught the tail end of a brighter flare in these JCMT sub-mm observations.

\subsection{The December 2015 mini-outburst}

Following the June 2015 outburst of V404 Cyg, renewed X-ray activity was detected from the system in December 2015 (MJD 57377, $\sim 189$ d after the first detection of the June outburst; Beardmore et al. 2015; Lipunov et al. 2015; Malyshev et al. 2015; Trushkin et al. 2015; Motta et al. 2016). Recent work (Muñoz-Darias et al. 2017; Kajava et al. 2018) has shown that while this 'mini-outburst' was in general fainter across all sampled frequencies when compared to the June outburst, it showed similar phenomenology; strong flaring activity, fast accretion disc wind, highly variable column density.

This mini-outburst phenomenon has been seen in other BHXB sources following bright outbursts (e.g. GRO J0422 + 32, XTE J1650-500, MAXI J1659-152, GRS 1739-278; Yan \& Yu 2017 and references within). However, with the exception of one source (Swift J1753.5-0127; Plotkin et al. 2017b), these mini-outbursts have only been monitored at X-ray and optical frequencies.

Muñoz-Darias et al. (2017) present AMI radio monitoring of this mini-outburst period, where they detect flaring radio emission for a $10 \mathrm{~d}$ period following the mini-outburst detection. This flaring period ended with a large radio flaring episode on December $31 /$ January 1, after which the radio emission began to decay (similar to the final radio flare on June 26 in the main outburst). We obtained JCMT SCUBA-2 sub-mm observations on January 1 and 2 , in which we observe a decreasing flux density trend in both the time-resolved measurements on January 1 and between the two JCMT epochs (see Fig. 10). This trend, combined with the timing of our measurements near the large radio flare, suggests that we may have caught the tail end of a brighter flare in our JCMT observations. Radio and sub-mm flaring accompanied repeated jet ejection events during the main June outburst (Tetarenko et al. 2017 and Fig. 3). Therefore, our measurements corroborate the theory suggested by Muñoz-Darias et al. (2017), which despite the fainter nature of the mini-outburst, V404 Cyg was still launching discrete jet ejecta during this time period, and in turn, jet ejecta are not exclusive to the highest luminosity states in V404 Cyg.

\section{SUMMARY}

In this paper, we present the results of our multifrequency monitoring observations of the 2015 outburst of the BHXB V404 Cyg. We observed the source at radio and $\mathrm{mm} / \mathrm{sub}-\mathrm{mm}$ frequencies, with the VLA, AMI, SMA, JCMT, and NOEMA, and collected publicly available OIR, UV, and X-ray measurements to supplement our coverage. With this well-sampled data set, we created detailed diagnostics of the jet emission in V404 Cyg, tracking the spectral and variability properties throughout different stages of the outburst (e.g. time-resolved light curves and spectral indices, broad-band spectra, CCFs, variability amplitude measurements).

Using these diagnostics, we find that the jet emission was originally dominated by emission from discrete jet ejecta during the brightest stages of the outburst. These ejecta appeared to become fainter, slower, less frequent, and less energetic, before the emission abruptly (over 1-2 d) transitioned to being dominated by a compact jet. While the broad-band spectrum of this compact jet showed very little evolution throughout the outburst decay (despite the intensity of the jet emission fading by an order of magnitude), the emission still remained intermittently variable at $\mathrm{mm} / \mathrm{sub}-\mathrm{mm}$ frequencies. Further, through phenomenological modelling of the broad-band emission from this compact jet, we directly detect the optically thick to thin synchrotron jet spectral break in the near-IR and optical bands $\left(\sim 2-5 \times 10^{14} \mathrm{~Hz}\right)$ and postulate that the compact jet could have been significantly contributing to the X-ray emission observed during the outburst decay.

Additionally, we compared the radio jet emission throughout the 2015 and previous 1989 outbursts. While the radio jet emission in both outbursts shows many similarities (e.g. peak flux and spectral index evolution), we show that the radio emission in the 2015 outburst decayed significantly ( $\sim 10$ times) faster than in the 1989 outburst. We postulate that, given the robust disc-jet coupling relationship found between these two outbursts (Plotkin et al. 2017a), this difference could indicate that the average mass accretion rate dropped (possibly due to the strong accretion disc wind) much quicker following the peak of the 2015 outburst, when compared to the 1989 outburst.

Lastly, we report on sub-mm observations during the December 2015 mini-outburst of V404 Cyg. These sub-mm observations display a decreasing flux trend, which most likely samples the tail end of a larger flaring episode. As sub-mm flaring coincided with jet ejection episodes during the main outburst, these observations support previous claims (Muñoz-Darias et al. 2017) that, similar to the main outburst, the source was most likely launching powerful jet ejecta during this period of renewed activity.

Overall, our work here demonstrates the importance of simultaneous, multifrequency, time-resolved observations to fully understand the rapidly evolving jet sources in BHXBs.

\section{ACKNOWLEDGEMENTS}

The authors thank the anonymous referee for constructive feedback that improved the manuscript. We wish to sincerely thank all the National Radio Astronomy Observatory, SMA, JCMT, and IRAM NOEMA staff that supported us during this observing campaign. The authors also offer a special thanks to the IRAM NOEMA Director, Karl-Fredrich Schuster, for granting our NOEMA DDT request. AJT thanks Craig Heinke and Bailey Tetarenko for useful discussions on accretion disc contributions in broad-band spectra of X-ray binaries. AJT is supported by an Natural Sciences and Engineering Research Council of Canada (NSERC) Post-Graduate 
Doctoral Scholarship (PGSD2-490318-2016). AJT and GRS are supported by NSERC Discovery Grants. JCAMJ is the recipient of an Australian Research Council Future Fellowship (FT140101082). SBM acknowledges support from VICI grant Nr. 639.043.513/520, funded by the Netherlands Organisation for Scientific Research (NWO). TDR acknowledges support from the NWO Veni Fellowship, grant number 639.041.646. DA acknowledges support from the Royal Society. This work is based on observations carried out under project numbers S15DE and D15AB with the IRAM NOEMA Interferometer. IRAM is supported by INSU/CNRS (France), MPG (Germany), and IGN (Spain). The National Radio Astronomy Observatory is a facility of the National Science Foundation operated under cooperative agreement by Associated Universities, Inc. We thank the engineers and staff of the Mullard Radio Astronomy Observatory for maintenance and operation of AMI, which is supported by the Universities of Cambridge and Oxford. The AMI telescope acknowledges support from the European Research Council under grant ERC-2012-StG-307215 LODESTONE. The SMA is a joint project between the Smithsonian Astrophysical Observatory and the Academia Sinica Institute of Astronomy and Astrophysics, and is funded by the Smithsonian Institution and the Academia Sinica. The JCMT is operated by the East Asian Observatory on behalf of The National Astronomical Observatory of Japan; Academia Sinica Institute of Astronomy and Astrophysics; the Korea Astronomy and Space Science Institute; the Operation, Maintenance and Upgrading Fund for Astronomical Telescopes and Facility Instruments, budgeted from the Ministry of Finance (MoF) of China and administrated by the Chinese Academy of Sciences (CAS), as well as the National Key R\&D Program of China (No. 2017YFA0402700). Additional funding support is provided by the Science and Technology Facilities Council of the United Kingdom and participating universities in the United Kingdom and Canada. The authors also wish to recognize and acknowledge the very significant cultural role and reverence that the summit of Mauna Kea has always had within the indigenous Hawaiian community. We are most fortunate to have the opportunity to conduct observations from this mountain. We acknowledge with thanks the variable star observations from the AAVSO International Database contributed by observers worldwide and used in this research.

\section{REFERENCES}

Akritas M. G., Bershady M. A., 1996, ApJ, 470, 706

Alexander T., 1997, Maoz D., Sternberg A., Leibowitz E. M, eds, Astronomical Time Series. p. 163, Kluwer Academic, Dordrecht, the Netherlands Alexander T., 2013, preprint(arXiv:1302.1508)

Arkharov A. et al., 2015, ATel, 7760, 1

Barthelmy S., D'Ai' A., D'Avanzo P., Krimm H., Lien A. Y., Marshall F. E., Maselli A., Siegel M. H., 2015, GCN Circular, 17929, 1

Beardmore A. P., Page K. L., Kuulkers E., 2015, ATel, 8455, 1

Beardmore A. P., Willingale R., Kuulkers E., Altamirano D., Motta S. E., Osborne J. P., Page K. L., Sivakoff G. R., 2016, MNRAS, 462, 1847

Belloni T., 2010, in Belloni T. M., ed., The Jet Paradigm - From Microquasars to Quasars, Lecture Notes in Physics 794. Springer, Berlin/Heidelberg

Bernardini F., Russell D. M., Shaw A. W., Lewis F., Charles P. A., Koljonen K. I. I., Lasota J. P., Casares J., 2016, ApJ, 818, L5

Blandford R. D., Königl A., 1979, ApJ, 232, 34

Brocksopp C., Corbel S., Tzioumis A., Broderick J. W., Rodriguez J., Yang J., Fender R. P., Paragi Z., 2013, MNRAS, 432, 931

Cardelli J., Clayton G., Mathis J., 1989, ApJ, 345, 245

Carrasco L., Recillas E., Porras A., Leon-Tavares J., Chavushyan V., Carraminana A., 2015, ATel, 7751, 1
Casares J., Charles P. A., Naylor T., Pavlenko E. P., 1993, MNRAS, 265, 834

Casella P. et al., 2010, MNRAS, 404, L21

Chandra P., Kanekar N., 2017, ApJ, 846, 111

Chapin E., Berry D., Gibb A., Jenness T., Scott D., Tilanus R., Economou F., Holland W., 2013, MNRAS, 430, 2545

Chaty S., Dubus G., Raichoor A., 2011, A\&A, 529, A3

Corbel S., Fender R., 2002, ApJ, 573, L35

Corbel S. et al., 2001, ApJ, 554, 43

Corbel S., Fender R. P., Tzioumis A. K., Tomsick J. A., Orosz J. A., Miller J. M., Wijnands R., Kaaret P., 2002, Science, 298, 196

Corbel S., Fender R. P., Tomsick J. A., Tzioumis A. K., Tingay S., 2004, ApJ, 617, 1272

Corbel S., Kording E., Kaaret P., 2008, MNRAS, 389, 1697

Coriat M. et al., 2011, MNRAS, 414, 677

Davies M. L. et al., 2009, MNRAS, 400, 984

Edelson R. A., Krolik J. H., 1988, ApJ, 333, 646

Falcke H., Biermann P., 1995, A\&A, 293, 665

Fender R., 2001, MNRAS, 322, 31

Fender R. et al., 1999, ApJ, 519, L165

Fender R., Belloni T., Gallo E., 2004, MNRAS, 355, 1105

Ferrigno C. et al., 2015, ATel, 7662, 1

Foreman-Mackey D., Hogg D. W., Lang D., Goodman J., 2013, PASP, 125, 306

Gallo E., Fender R., Pooley G. G., 2003a, MNRAS, 344, 60

Gallo E., Fender R. P., Pooley G., 2003b, MNRAS, 344, 60

Gallo E., Fender R., Hynes R., 2005, MNRAS, 356, 1017

Gallo E., Migliari S., Markoff S., Tomsick J., Bailyn C., Berta S., Fender R., Miller-Jones J., 2007, ApJ, 670, 600

Gallo E. et al., 2014, MNRAS, 445, 290

Gallo E., Degenaar N., van den Eijnden J., 2018, MNRAS, 478, L132

Gandhi P., Littlefair S., Hardy L., Dhillon V., Marsh T., Shaw A. W., 2015, ATel, 7686, 1

Gandhi P. et al., 2017, Nature Astron., 1, 859

Gaskell C. M., Peterson B. M., 1987, ApJS, 65, 1

Gazeas K., Vasilopoulos G., Petropoulou M., Sapoutntzis K., 2015, ATel, 7650,1

Han X., Hjellming R. M., 1992, ApJ, 400, 304

Heinz S., Corrales L., Smith R., Brandt W. N., Jonker P. G., Plotkin R. M., Neilsen J., 2016, ApJ, 825, 15

Hjellming R., Rupen M., 1995, Nature, 375, 464

Hjellming R., Rupen M. P., Mioduszewski A., Narayan R., 2000, ATel, 54, 1

Holland W. et al., 2013, MNRAS, 430, 2513

Hynes R., Haswell C., Chaty S., Shrader C., Cui W., 2002, MNRAS, 331, 169

Hynes R., Bradley C., Rupen M. P., Gallo E., Fender R., Casares J., Zurita C., 2009, MNRAS, 399, 2239

Hynes R. I., 2005, ApJ, 623, 1026

Jamil O., Fender R., Kaiser C., 2010, MNRAS, 401, 394

Kafka S., 2018, Observations from the AAVSO International Database. Available at: https://www.aavso.org

Kaiser C., 2006, MNRAS, 367, 1083

Kajava J. J. E., Motta S. E., Sánchez-Fernández C., Kuulkers E., 2018, A\&A, 616, A129

Khargharia J., Froning C., Robinson E. L., 2010, ApJ, 716, 1105

Kimura M. et al., 2016, Nature, 529, 54

Koljonen K. et al., 2015, ApJ, 814, 139

Kuulkers E., 2015, ATel, 7758, 1

Kuulkers E., Fender R. P., Spencer R., Davis R., Morison I., 1999, MNRAS, 306, 919

Kuulkers E., Motta S., Kajava J., Homan J., Fender R., Jonker P., 2015, ATel, 7647, 1

Lipunov V. et al., 2015, ATel, 8453, 1

Maitra D., Scarpaci J. F., Grinberg V., Reynolds M. T., Markoff S., Maccarone T. J., Hynes R. I., 2017, ApJ, 851, 148

Makino F., 1989, IAU Circ., 4782, 1 
Malyshev D., Savchenko V., Ferrigno C., Bozzo E., Kuulkers E., 2015, ATel, 8458,1

Malzac J., 2014, MNRAS, 443, 229

Malzac J., Belloni T., Spruit H., Kanbach G., 2003, A\&A, 407, 335

Markoff S., Falcke H., Fender R., 2001, A\&A, 372, L25

Markoff S., Nowak M., Wilms J., 2005, ApJ, 635, 1203

McMullin J. P., Waters B., Schiebel D., Young W., Golap K., 2007, in Shaw R. A., Hill F., Bell D. J., eds, ASP Conf. Ser. Vol. 376, Astronomical Data Analysis Software and Systems XVI. Astron. Soc. Pac., San Francisco, p. 127

Miller-Jones J. C. A., Jonker P. G., Dhawan V., Brisken W., Rupen M. P., Nelemans G., Gallo E., 2009, ApJ, 706, L230

Miller-Jones J. C. A. et al., 2012, MNRAS, 421, 468

Miller-Jones J. C. A., Blundell K. M., Rupen M. P., Mioduszewski A. J., Duffy P., Beasley A. J., 2004, ApJ, 600, 368

Mirabel I. F., Rodríguez L. F., 1994, Nature, 371, 46

Mooley K., Fender R., Anderson G., Staley T., Kuulkers E., Rumsey C., 2015, ATEL, 7658, 1

Motta S., Beardmore A., Oates S., Sanna N. P. M. K. A., Kuulkers E., Kajava J., Sanchez-Fernanedz C., 2015a, ATel, 7665, 1

Motta S., Beardmore A., Oates S., Sanna N. P. M. K. A., Kuulkers E., Kajava J., Sanchez-Fernanedz C., 2015b, ATel, 7666, 1

Motta S. et al., 2016, ATel, 8510, 1

Motta S. E., Kajava J. J. E., Sánchez-Fernández C., Giustini M., Kuulkers E., 2017a, MNRAS, 468, 981

Motta S. E. et al., 2017b, MNRAS, 471, 1797

Muno M., Mauerhan J., 2006, ApJ, 648, L135

Munoz-Darias T., Casares J., Mata Sanchez D., Fender R. P., Armas Padilla M., 2016, Nature, 534, 75

Muñoz-Darias T. et al., 2017, MNRAS, 465, L124

Negoro H. et al., 2015, ATel, 7579, 1

Oates S. R., Motta S., Kuulkers E., Kuin N. P. M., Altamirano D., Gandhi P., Knigge C., Kennea J. A., 2015, ATel, 7734, 1

Oosterbroek T. et al., 1997, A\&A, 321, 776

Pe'er A., Casella P., 2009, ApJ, 699, 1919

Pe'er A., Markoff S., 2012, ApJ, 753, 177

Perley R. A., Butler B. J., 2017, ApJS, 230, 7

Perrott Y. C. et al., 2013, MNRAS, 429, 3330

Plotkin R., Gallo E., Jonker P., 2013, ApJ, 773, 59

Plotkin R., Gallo E., Markoff S., Homan J., Jonker P., Miller-Jones J., Russell D., Drappeau S., 2015, MNRAS, 446, 4098

Plotkin R. M. et al., 2016, MNRAS, 456, 2707

Plotkin R. M. et al., 2017a, ApJ, 834, 19

Plotkin R. M. et al., 2017b, ApJ, 848, 92

Richter G., 1989, IBVS, 3362, 1
Rodriguez J., Cadolle Bel M., Alfonso-Garzon J. et al., 2015, A\&A, 581, L9

Rushton A. P. et al., 2016, MNRAS, 463, 628

Russell D., Fender R., Hynes R., Brocksopp C., Homan J., Jonker P., Buxton M., 2006, MNRAS, 371, 1334

Russell D., Maitra D., Dunn R., Markoff S., 2010, MNRAS, 405, 1759

Russell D. et al., 2013a, MNRAS, 429, 815

Russell T., Soria R., Miller-Jones J., Curran P., Markoff S., Russell D., Sivakoff G., 2014, MNRAS, 439, 1390

Russell T. D. et al., 2015, MNRAS, 450, 1745

Russell D. M., Miller-Jones J. C. A., Maccarone T. J., Yang Y. J., Fender R. P., Lewis F., 2011, ApJ, 739, L19

Russell D. M. et al., 2013b, ApJ, 768, L35

Sadler E. M. et al., 2006, MNRAS, 371, 898

Sari R., Piran T., Narayan R., 1998, ApJ, 497, L17

Shaw A., 2015, ATel, 7738, 1

Sivakoff G. R., Bahramian A., Altamirano D., Miller-Jones J. C. A., Russell D., 2015a, ATel, 7763, 1

Sivakoff G. R., Bahramian A., Heinke C., Tetarenko A., Knigge C., Bozzo E., Esposito V., Fender R., 2015b, ATel, 7788, 1

Terada K., Miyamoto S., Kitamoto S., Egoshi W., 1994, PASJ, 46, 677

Tetarenko A., Sivakoff G., Gurwell M., Petitpas G., Wouterloot J., MillerJones J., 2015b, ATel, 7661, 1

Tetarenko A., Sivakoff G., Young K., Wouterloot J., Miller-Jones J., 2015c, ATel, 7708, 1

Tetarenko A., Sivakoff G. R., Miller-Jones J., Coulson I., 2016, ATel, 8499, 1

Tetarenko A. J. et al., 2015a, ApJ, 805, 30

Tetarenko A. J. et al., 2017, MNRAS, 469, 3141

Tingay S. J. et al., 1995, Nature, 374, 141

Trushkin S. A., Nizhelskij N. A., Tsybulev P., 2015, ATEL, 8454, 1

van der Horst A. et al., 2013, MNRAS, 436, 2625

van der Laan H., 1966, Nature, 5054, 1131

Vaughan S., Edelson R., Warwick R. S., Uttley P., 2003, MNRAS, 345, 1271

Verner D. A., Ferland G. J., Korista K. T., Yakovlev D. G., 1996, ApJ, 465, 487

Vincentelli F. M. et al., 2018, MNRAS, 477, 4524

Wagner R. M., Starrfield S. G., Cassatella A., Hurst G. M., Mobberley M., Marsden B. G., 1989, IAU Circ., 4783, 1

Wilms J., Allen A., McCray R., 2000, ApJ, 542, 914

Yan Z., Yu W., 2017, MNRAS, 470, 4298

Zwart J. T. L. et al., 2008, MNRAS, 391, 1545

Zycki P., Done C., Smith D., 1999b, MNRAS, 309, 561

Zycki P. T., Done C., Smith D. A., 1999a, MNRAS, 305, 231 


\section{APPENDIX A: OBSERVATION SET-UP}

In this section, we provide details on the correlator and array set-up of all of our radio through mm/sub-mm interferometric observations: VLA (Table A1), NOEMA (Table A2), and SMA (Table A3).

Table A1. VLAobservations summary.

\begin{tabular}{|c|c|c|c|c|c|}
\hline $\begin{array}{l}\text { Date } \\
(2015)\end{array}$ & MJD & Sub-array & $\begin{array}{l}\text { Scans } \\
\text { (UTC) }\end{array}$ & $\operatorname{Band}(\mathrm{s})^{a}$ & $\begin{array}{c}\text { Number of } \\
\text { antennas }\end{array}$ \\
\hline \multirow[t]{2}{*}{ July 02} & 57205 & A & 10:02:14-14:01:32 & $C-K-C$ & 14 \\
\hline & & B & 10:05:14-14:01:32 & $K-C-K$ & 13 \\
\hline \multirow[t]{3}{*}{ July 10} & 57213 & A & $12: 03: 02-13: 01: 50$ & C & 8 \\
\hline & & $\mathrm{B}$ & $12: 04: 02-13: 01: 52$ & $L$ & 9 \\
\hline & & $\mathrm{C}$ & $12: 02: 02-13: 01: 50$ & $\mathrm{Ku}$ & 9 \\
\hline \multirow[t]{3}{*}{ July 11} & 57214 & A & $13: 29: 52-14: 27: 40$ & $L$ & 9 \\
\hline & & $\mathrm{B}$ & $13: 28: 52-14: 27: 40$ & $C$ & 8 \\
\hline & & $\mathrm{C}$ & $13: 27: 52-14: 27: 40$ & $\mathrm{Ku}$ & 9 \\
\hline \multirow[t]{3}{*}{ July 12} & 57215 & A & $12: 04: 02-13: 01: 52$ & $L$ & 8 \\
\hline & & $\mathrm{B}$ & $12: 03: 02-13: 01: 50$ & $C$ & 9 \\
\hline & & $\mathrm{C}^{b}$ & - & $\mathrm{Ku}$ & 8 \\
\hline
\end{tabular}

Notes. ${ }^{a}$ This column indicates the frequency bands observed with each sub-array. When multiple bands are present, the entry in this column indicates the temporal sequence with which the bands were observed by the sub-array.

${ }^{b}$ A technical error occurred during observing and no data was taken for this sub-array.

Table A2. NOEMAobservations summary.

\begin{tabular}{lccccc}
\hline $\begin{array}{l}\text { Date } \\
(2015)\end{array}$ & MJD & Array config. & $\begin{array}{c}\text { Scans } \\
(\text { UTC) }\end{array}$ & $\begin{array}{c}\text { Band } \\
(\mathrm{mm})\end{array}$ & $\begin{array}{c}\text { Number of } \\
\text { antennas }\end{array}$ \\
\hline June 26 & 57199 & 6Dq-E03+E12 & $22: 24: 02-23: 12: 58$ & 3 & 6 \\
June 27 & 57200 & 6Dq-E03+E12 & $00: 15: 35-02: 42: 31$ & 2 & 6 \\
June 27/28 & $57200 / 57201$ & 6Dq-E03+E12 & $23: 17: 37-03: 05: 34$ & 2 & 6 \\
June 29 & 57202 & 6 Dq & $22: 13: 50-23: 02: 47$ & 3 & 6 \\
June 30 & 57203 & 6Dq & $00: 14: 51-02: 55: 10$ & 2 & 6 \\
July 12 & 57215 & 6Dq & $22: 03: 27-22: 52: 23$ & 3 & 6 \\
July 12/13 & $57215 / 57216$ & 6Dq & $23: 45: 19-02: 12: 09$ & 2 & 6
\end{tabular}

Table A3. SMA observationssummary ${ }^{a}$.

\begin{tabular}{|c|c|c|c|c|c|c|c|}
\hline $\begin{array}{l}\text { Date } \\
(2015)\end{array}$ & MJD & Array config. & $\begin{array}{l}\text { Scans } \\
\text { (UTC) }\end{array}$ & $\begin{array}{c}\text { Total bandwidth } \\
\text { (GHz) }\end{array}$ & Correlator & $\begin{array}{c}\text { Correlator set-up } \\
\left(N_{\mathrm{spw}}, N_{\mathrm{C}}, \Delta v \mathrm{MHz}\right)^{b}\end{array}$ & $\begin{array}{c}\text { Number of } \\
\text { antennas }\end{array}$ \\
\hline June 16 & 57189 & Sub-compact & $17: 22: 33-18: 40: 43$ & 8.32 & ASIC+SWARM & $(48,128,0.8125)+(2,128,13)^{c}$ & 7 \\
\hline July 01 & 57204 & Compact & $07: 50: 28-17: 18: 53$ & 2.080 & ASIC & $(20,32,3.250)$ & 6 \\
\hline July 02 & 57205 & Compact & $09: 26: 57-14: 30: 42$ & 2.496 & ASIC & $(24,32,3.250)^{d}$ & 6 \\
\hline
\end{tabular}

Notes. ${ }^{a}$ Our SMA project is a ToO programme, with highly constrained start times, needed to obtain simultaneous observations with other facilities. Therefore, due to the continuum nature of our observations, our programme is often run immediately before or after other SMA observing programmes, which results in the wide variety of correlator set-ups seen here.

${ }^{b}$ The correlator set-up, number of spectral windows, number of channels, and channel width for each of the two side bands.

${ }^{c}$ The SWARM correlator had a fixed resolution of $101.6 \mathrm{kHz}$ per channel with 16383 channels for each SWARM spectral window. Given the continuum nature of these observations, we performed spectral averaging to yield $12813-\mathrm{MHz}$ channels in both SWARM spectral windows (matching the number of channels in the ASIC spectral windows) and to make it easier to combine ASIC and SWARM data.

${ }^{d}$ Three of the 24 spectral windows were set up with a higher spectral resolution in this observation; $5120.203-\mathrm{MHz}$ channels. As such, we spectrally averaged these channels to match the other spectral windows with 32 3.250-MHz channels. 


\section{APPENDIX B: OBSERVATIONAL DATA}

In this section, we provide data tables of all the day-time-scale multifrequency photometry measurements presented in this work: radio (Table B1), mm/sub-mm (Table B2), and OIR/UV/X-ray (Table B3).

Table B1. Flux densitiesof V404 Cyg at radio frequencies.

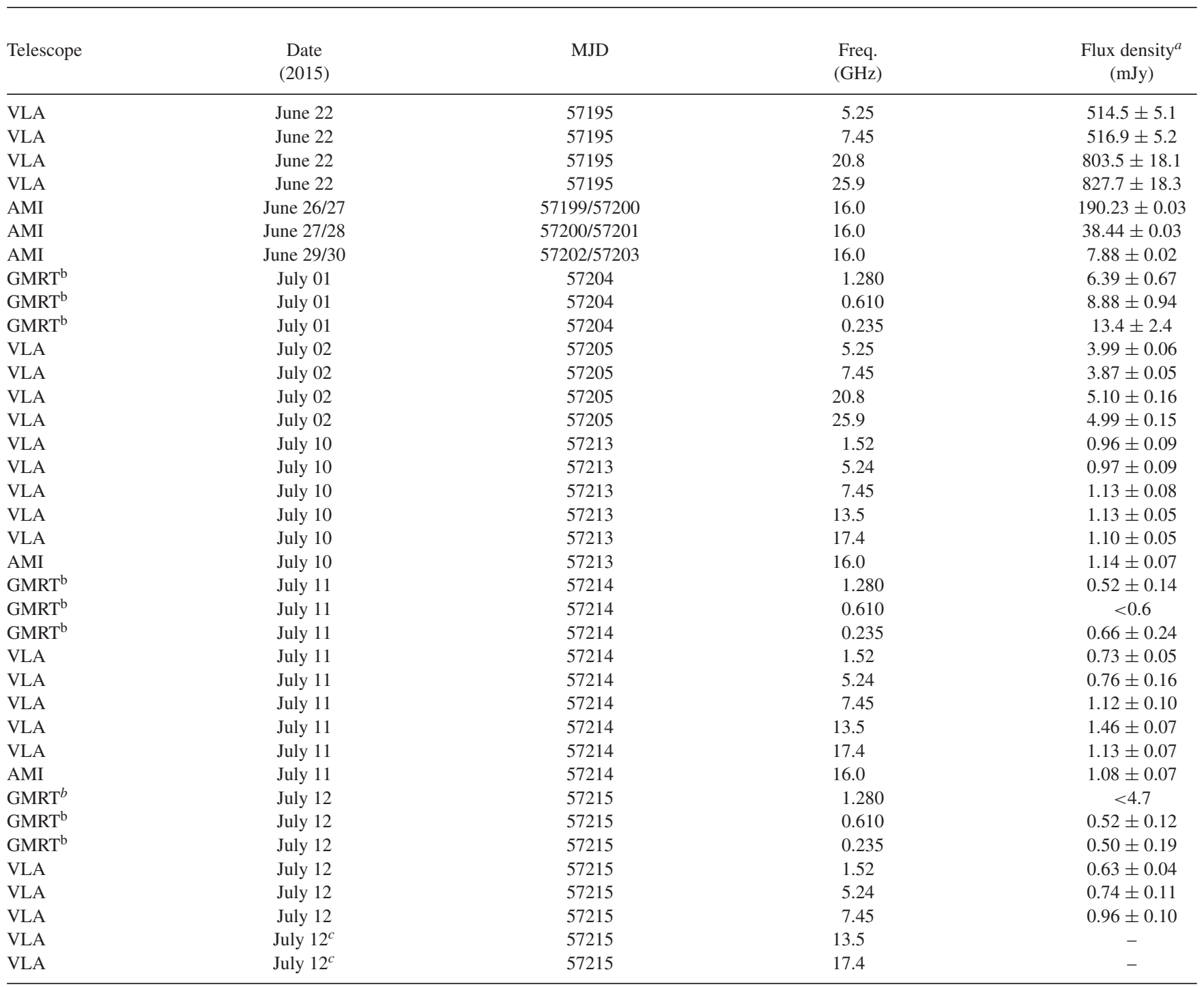

${ }^{a}$ The VLA measurements include the standard VLA systematic errors.

${ }^{b}$ Giant Metrewave Radio Telescope (GMRT) data are taken from Chandra \& Kanekar (2017).

${ }^{c}$ A technical error occurred during observing, and thus no data was taken for this sub-array. 
Table B2. Flux densitiesof V404 Cyg at mm/sub-mm frequencies.

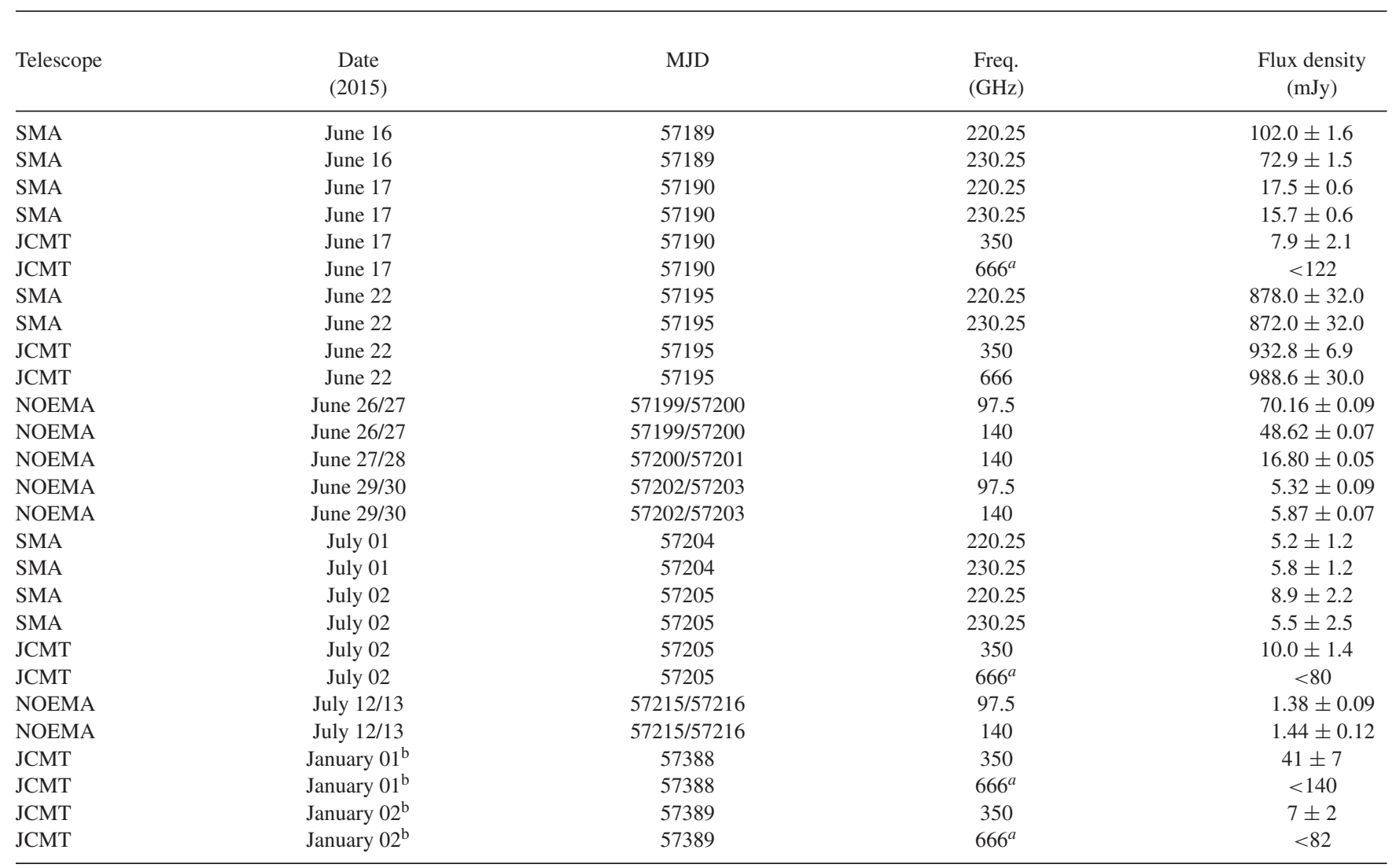

${ }^{a}$ Note that data in the $666 \mathrm{GHz}(450 \mu \mathrm{m})$ band was obtained simultaneously with the $350 \mathrm{GHz}(850 \mu \mathrm{m})$ band, but the source was not always significantly detected at $666 \mathrm{GHz}$. The values reported here represent $3 \sigma$ upper limits.

${ }^{\mathrm{b}}$ These data were taken during the late December 2015/January 2016 mini-outburst of V404 Cyg. 
Table B3. Flux densities of V404Cyg at IR/optical/UV/X-ray frequencies.

\begin{tabular}{|c|c|c|c|c|c|}
\hline Band $^{a}$ & $\begin{array}{l}\text { Date } \\
(2015)\end{array}$ & MJD & $\begin{array}{l}\text { Freq. } \\
(\mathrm{GHz})\end{array}$ & $\begin{array}{l}\text { Flux density } \\
\qquad(\mathrm{mJy})\end{array}$ & $\operatorname{Ref}^{b}$ \\
\hline$R$ & June 16 & 57189 & $4.56 \times 10^{5}$ & $212.2 \pm 22.9$ & 5 \\
\hline$J$ & June $26 / 27$ & $57199 / 57200$ & $2.45 \times 10^{5}$ & $46.6 \pm 4.9$ & 1 \\
\hline$H$ & June $26 / 27$ & $57199 / 57200$ & $1.84 \times 10^{5}$ & $50.5 \pm 5.0$ & 1 \\
\hline K & June $26 / 27$ & $57199 / 57200$ & $1.37 \times 10^{5}$ & $37.6 \pm 2.9$ & 1 \\
\hline$U$ & June $26 / 27$ & $57199 / 57200$ & $8.65 \times 10^{5}$ & $25.7 \pm 1.0$ & 2 \\
\hline$J$ & June $27 / 28$ & $57200 / 57201$ & $2.45 \times 10^{5}$ & $98.7 \pm 8.6$ & 3 \\
\hline$H$ & June $27 / 28$ & $57200 / 57201$ & $1.84 \times 10^{5}$ & $77.9 \pm 8.5$ & 3 \\
\hline K & June $27 / 28$ & $57200 / 57201$ & $1.37 \times 10^{5}$ & $67.4 \pm 6.6$ & 3 \\
\hline V & June $27 / 28$ & $57200 / 57201$ & $5.48 \times 10^{5}$ & $19.1 \pm 1.9$ & 2 \\
\hline$B$ & June $27 / 28$ & $57200 / 57201$ & $6.82 \times 10^{5}$ & $16.1 \pm 2.4$ & 2 \\
\hline$U V 1$ & June $27 / 28$ & $57200 / 57201$ & $1.15 \times 10^{6}$ & $2.2 \pm 9.1$ & 2 \\
\hline$J$ & June $29 / 30$ & $57202 / 57203$ & $2.45 \times 10^{5}$ & $30.7 \pm 0.6$ & 4 \\
\hline$H$ & June $29 / 30$ & $57202 / 57203$ & $1.84 \times 10^{5}$ & $25.8 \pm 0.6$ & 4 \\
\hline K & June $29 / 30$ & $57202 / 57203$ & $1.37 \times 10^{5}$ & $22.3 \pm 0.5$ & 4 \\
\hline$I$ & June $29 / 30$ & $57202 / 57203$ & $3.72 \times 10^{5}$ & $30.9 \pm 2.7$ & 5 \\
\hline V & June $29 / 30$ & $57202 / 57203$ & $5.48 \times 10^{5}$ & $19.9 \pm 2.0$ & 2 \\
\hline$B$ & June $29 / 30$ & $57202 / 57203$ & $6.82 \times 10^{5}$ & $18.4 \pm 2.4$ & 2 \\
\hline$U$ & June $29 / 30$ & $57202 / 57203$ & $8.65 \times 10^{5}$ & $11.4 \pm 2.3$ & 2 \\
\hline$U V 1$ & June $29 / 30$ & $57202 / 57203$ & $1.15 \times 10^{6}$ & $12.7 \pm 10.7$ & 2 \\
\hline$U V 2$ & June $29 / 30$ & $57202 / 57203$ & $1.55 \times 10^{6}$ & $2.9 \pm 35.2$ & 2 \\
\hline$X R T$ & June 30 & 57203 & $1.21 \times 10^{9}$ & $\left(6.6_{-0.4}^{+0.5}\right) \times 10^{-3}$ & 6 \\
\hline V & July 1 & 57204 & $5.48 \times 10^{5}$ & $29.8 \pm 1.7$ & 2 \\
\hline$B$ & July 1 & 57204 & $6.82 \times 10^{5}$ & $20.4 \pm 1.9$ & 2 \\
\hline$U$ & July 1 & 57204 & $8.65 \times 10^{5}$ & $13.8 \pm 2.3$ & 2 \\
\hline$U V 1$ & July 1 & 57204 & $1.15 \times 10^{6}$ & $5.7 \pm 7.9$ & 2 \\
\hline$U V 2$ & July 1 & 57204 & $1.55 \times 10^{6}$ & $41.0 \pm 33.3$ & 2 \\
\hline$J$ & July 2 & 57205 & $2.45 \times 10^{5}$ & $29.9 \pm 2.6$ & 1 \\
\hline$H$ & July 2 & 57205 & $1.84 \times 10^{5}$ & $31.8 \pm 2.9$ & 1 \\
\hline K & July 2 & 57205 & $1.37 \times 10^{5}$ & $24.3 \pm 2.1$ & 1 \\
\hline$I$ & July 2 & 57205 & $3.72 \times 10^{5}$ & $42.5 \pm 4.0$ & 5 \\
\hline V & July 2 & 57205 & $5.48 \times 10^{5}$ & $19.6 \pm 2.1$ & 2 \\
\hline$B$ & July 2 & 57205 & $6.82 \times 10^{5}$ & $18.9 \pm 2.4$ & 2 \\
\hline$U$ & July 2 & 57205 & $8.65 \times 10^{5}$ & $14.5 \pm 3.1$ & 2 \\
\hline$U V 1$ & July 2 & 57205 & $1.15 \times 10^{6}$ & $7.3 \pm 9.7$ & 2 \\
\hline$X R T$ & July 2 & 57205 & $1.21 \times 10^{9}$ & $\left(4.7_{-03}^{+0.3}\right) \times 10^{-3}$ & 6 \\
\hline$I$ & July 10 & 57213 & $3.72 \times 10^{5}$ & $22.3 \pm 2.0$ & 8 \\
\hline$R$ & July 10 & 57213 & $4.56 \times 10^{5}$ & $23.4 \pm 0.4$ & 8 \\
\hline$V$ & July 10 & 57213 & $5.48 \times 10^{5}$ & $14.8 \pm 0.8$ & 8 \\
\hline$U$ & July 10 & 57213 & $8.65 \times 10^{5}$ & $5.1 \pm 0.6$ & 2 \\
\hline$U V 1$ & July 10 & 57213 & $1.15 \times 10^{6}$ & $6.1 \pm 1.3$ & 2 \\
\hline$X R T$ & July 10 & 57213 & $1.21 \times 10^{9}$ & $\left(1.0_{-0.1}^{+0.2}\right) \times 10^{-3}$ & 6 \\
\hline I & July 11 & 57214 & $3.72 \times 10^{5}$ & $22.3 \pm 2.0$ & 8 \\
\hline$R$ & July 11 & 57214 & $4.56 \times 10^{5}$ & $21.1 \pm 1.1$ & 8 \\
\hline$V$ & July 11 & 57214 & $5.48 \times 10^{5}$ & $15.0 \pm 0.4$ & 8 \\
\hline Chandra & July 11 & 57214 & $1.21 \times 10^{9}$ & $\left(1.2_{-0.01}^{+0.02}\right) \times 10^{-3}$ & 7 \\
\hline$I$ & July 12 & 57214 & $3.72 \times 10^{5}$ & $20.1 \pm 1.0$ & 8 \\
\hline$R$ & July 12 & 57214 & $4.56 \times 10^{5}$ & $17.6 \pm 1.6$ & 8 \\
\hline V & July 12 & 57214 & $5.48 \times 10^{5}$ & $13.7 \pm 0.8$ & 8 \\
\hline
\end{tabular}

Notes. ${ }^{a}$ This column displays the filters/instruments used for each observation: $U$ (UVOT $U$ band), $B$ (UVOT $B$ band), $V$ (UVOT/optical $V$ band), $U V 1$ (UVOT $U V W 1$ band), $U V 2$ (UVOT $U V W 2$ band), $R$ (optical $R$ band), $I$ (opticallband), $J$ (infrared $J$ band), $H$ (infrared $H$ band), $K$ (infrared $K$ band), Swift X-ray Telescope (XRT;0.5-10 keV), Chandra $(0.5-10 \mathrm{keV})$. Data shown in this table have been de-reddened (when required) using the prescription in Cardelli et al. (1989), with an $E(B-V)=1.3 \pm 0.2$ (Casares et al. 1993).

${ }^{b} 1$ : Arkharov et al. (2015); 2: Oates et al. (2015); 3: Shaw (2015); 4: Carrasco et al. (2015); 5: Kimura et al. (2016); 6: Sivakoff et al. (2015b); 7: Plotkin et al. (2017a); 8: AAVSO, Kafka (2018); Observations from the AAVSO International Database, https://www.aavso.org. 


\section{APPENDIX C: JET MODELLING RESULTS}

In this section, we show additional figures pertaining to our light-curve modelling. Fig. C1 displays an alternate version of Fig. 6, in which we decompose the total jet model into the individual approaching and receding components. Fig. C2 displays a zoomed-in version of Fig. 3 , where we also indicate the ejection times predicted from our modelling.

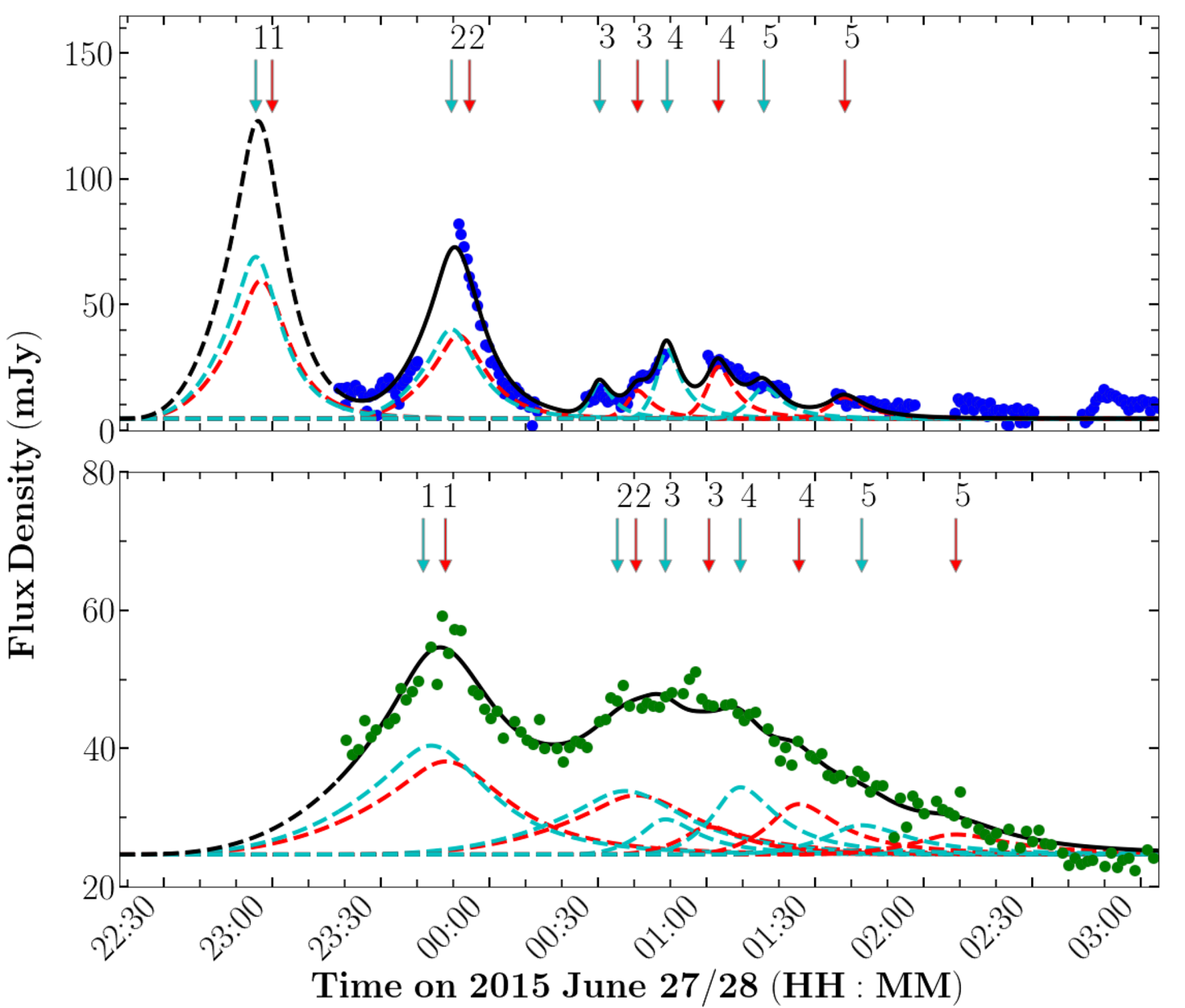

Figure C1. Radio (AMI $16 \mathrm{GHz}$; bottom panel) and mm/sub-mm (NOEMA $140 \mathrm{GHz}$; top panel) light curves of V404 Cyg on $2015 \mathrm{June} 27 / 28$ (MJD 57200/57201). In both panels, the black lines represent our predicted best-fitting jet model at each frequency, and the dotted lines indicate the approaching (cyan) and receding (red) components of the individual ejection events. The arrows at the top of each panel (cyan for approaching, red for receding) identify which flares correspond to which ejection number from Table 1. 


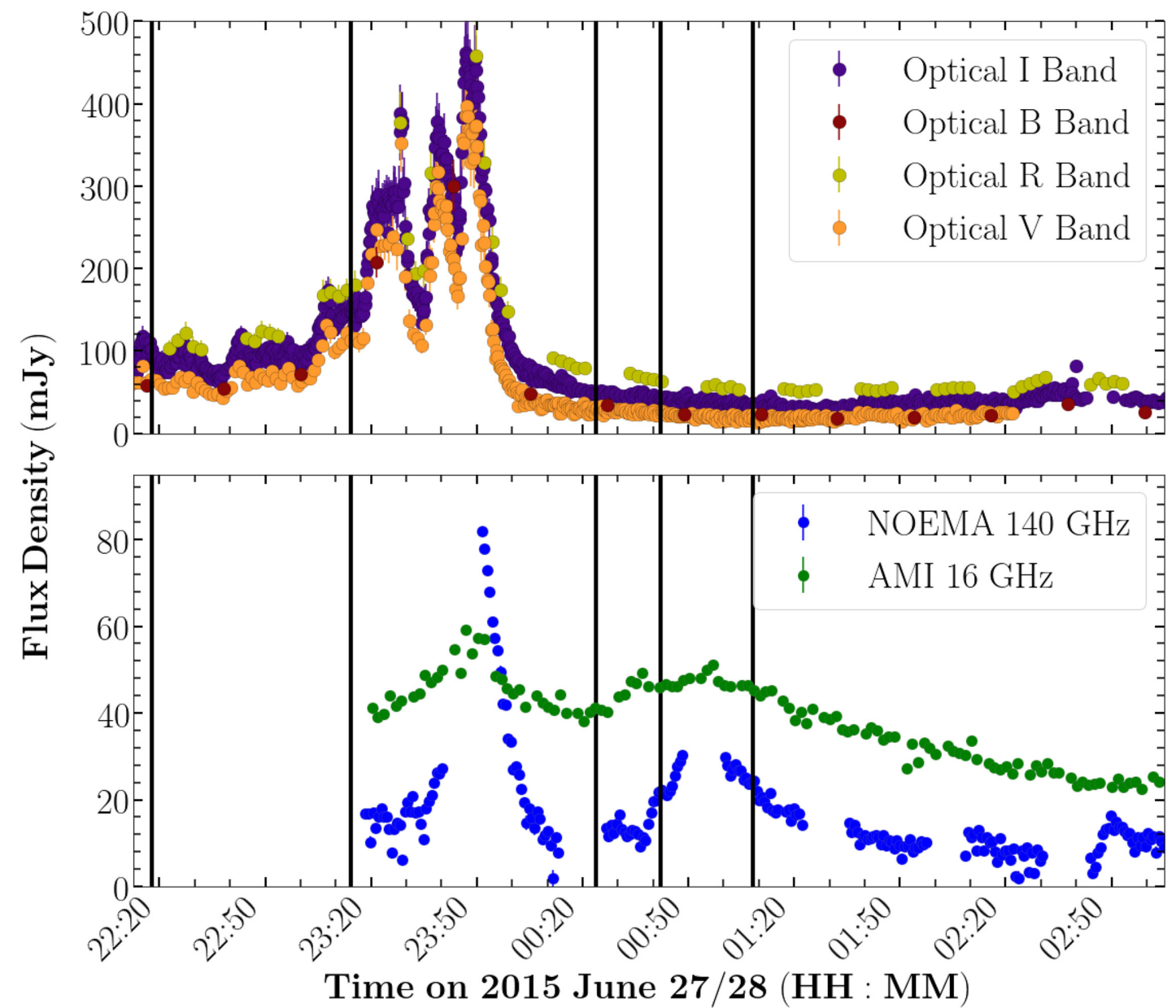

Figure C2. Zoomed-in version of Fig. 3, showing the optical (I, B. R. and V bands; top panel), as well as radio (AMI 16 GHz) and mm/sub-mm (NOEMA $140 \mathrm{GHz}$; bottom panel) light curves of V404 Cyg on 2015 June 27/28 (MJD 57200/57201). The vertical black lines indicate the predicted ejection times for each jet ejection event we model (see Table 1). While ejection 2 coincides with the beginning of a large optical flaring complex, the ejection times for later events do not seem to have optical counterparts (similar to what was seen during our previous modelling of flaring on MJD 57195; Tetarenko et al. 2017).

This paper has been typeset from a $\mathrm{TE}_{\mathrm{E}} / \mathrm{L} \mathrm{T} \mathrm{E} \mathrm{X}$ file prepared by the author. 